\title{
Spectrum of Short- and Long-Term Brain Pathology and Long-Term Behavioral Deficits in Male Repeated Hypoxic Rats Closely Resembling Human Extreme Prematurity
}

\author{
Dorothy E. Oorschot ${ }^{1,3}$ Logan Voss, ${ }^{1,3 *}$ Matthew V. Covey, ${ }^{1,3 *}$ Liping Goddard, ${ }^{1,3}$ William Huang, ${ }^{1,3}$ Penny Birchall, ${ }^{1,3}$ \\ David K. Bilkey,,$^{2,3}$ and Sarah E. Kohe ${ }^{1,3}$ \\ ${ }^{1}$ Department of Anatomy, Otago School of Medical Sciences, ${ }^{2}$ Department of Psychology, and ${ }^{3}$ Brain Health Research Centre, University of Otago, Dunedin \\ 9054, New Zealand
}

\begin{abstract}
Brain injury in the premature infant is associated with a high risk of neurodevelopmental disability. Previous small-animal models of brain injury attributable to extreme prematurity typically fail to generate a spectrum of pathology and behavior that closely resembles that observed in humans, although they provide initial answers to numerous cellular, molecular, and therapeutic questions. We tested the hypothesis that exposure of rats to repeated hypoxia from postnatal day 1 (P1) to P3 models the characteristic white matter neuropathological injury, gray matter volume loss, and memory deficits seen in children born extremely prematurely. Male Sprague Dawley rats were exposed to repeated hypoxia or repeated normoxia from P1 to P3. The absolute number of pre-oligodendrocytes and mature oligodendrocytes, the surface area and $g$-ratio of myelin, the absolute volume of cerebral white and gray matter, and the absolute number of cerebral neurons were quantified stereologically. Spatial memory was investigated on a radial arm maze. Rats exposed to repeated hypoxia had a significant loss of (1) pre-oligodendrocytes at P4, (2) cerebral white matter volume and myelin at P14, (3) cerebral cortical and striatal gray matter volume without neuronal loss at P14, and (4) cerebral myelin and memory deficits in adulthood. Decreased myelin was correlated with increased attention deficit hyperactivity disorder-like hyperactivity. This new small-animal model of extreme prematurity generates a spectrum of short- and long-term pathology and behavior that closely resembles that observed in humans. This new rat model provides a clinically relevant tool to investigate numerous cellular, molecular, and therapeutic questions on brain injury attributable to extreme prematurity.
\end{abstract}

\section{Introduction}

Two percent of infants (currently 86,000 of all live births in the United States annually) are born extremely prematurely (22-28 weeks of gestation; Volpe, 2009). Because the survival rate of these infants is now $\sim 90 \%$ (Volpe, 2009), the fact that $50-70 \%$ will have behavioral and memory deficits later in life (Johnson, 2007 ) is a significant health problem. For example, the perinatal care costs alone for babies born extremely prematurely exceeds $\$ 18$ billion annually in the United States (Ment et al., 2009).

Received Jan. 20, 2012; revised May 24, 2013; accepted May 27, 2013.

Author contributions: D.E.O., L.V., M.V.C., L.G., D.K.B., and S.E.K. designed research; D.E.O., L.V., M.V.C., L.G., W.H., P.B., and S.E.K. performed research; D.E.O., L.V., M.V.C., L.G., W.H., P.B., and S.E.K. analyzed data; D.E.O., L.V., M.V.C., L.G., D.K.B., and S.E.K. wrote the paper.

This work was supported by the Otago Medical Research Foundation (D.E.O. and L.V.), the Strategic Research Fund of the Department of Anatomy (D.E.O. and L.G.), a Department of Anatomy Summer Studentship Award and Masters Postgraduate Scholarship (S.E.K.), and University of Otago PhD Scholarships (M.V.C. and S.E.K.). We gratefully acknowledge the clinical support of Prof. Barry Taylor (Department of Women's and Children's Health, University of Otago) during the initial development of the animal model, the technical support of Michael Davis and of Sharon Lequeux and Gillian Grayston of the Otago Centre for Electron Microscopy, and the support of Dr. Rachel Sizemore and Robbie McPhee in creating the figures.

*L.V. and M.V.C. contributed equally to this research.

Correspondence should be addressed to Assoc. Prof. Dorothy Oorschot, Department of Anatomy, Otago School of Medical Sciences, University of Otago, P.0. Box 913, Dunedin 9054, New Zealand. E-mail: dorothy.oorschot@anatomy.otago.ac.nz.

DOI:10.1523/JNEUROSCI.0342-12.2013

Copyright $\odot 2013$ the authors $\quad 0270-6474 / 13 / 3311863-15 \$ 15.00 / 0$
Because rates of prematurity have not declined in the past decade, this major burden of neurological disability can be expected to continue (Volpe et al., 2011). Males, in particular, are vulnerable (Venderhus et al., 2010).

The cerebral neuropathology most frequently described in extremely premature infants consists of preferential white matter damage, termed diffuse periventricular leukomalacia (PVL; Volpe et al., 2011). Diffuse PVL may occur via exposure to repeated bouts of hypoxia because extremely premature infants often experience repeated bouts of sublethal hypoxia in neonatal intensive care units despite medical intervention (Poets et al., 1995; Lou, 1996). Diffuse PVL is initially characterized by a significant loss of O4-positive pre-oligodendrocytes (Volpe et al., 2011). Later sequelae include decreased white matter volume and hypomyelination in the cerebrum (Volpe, 2009) that persists long term (Ment et al., 2009). A decreased volume of the cerebral gray matter, without cortical or striatal neuronal loss, often accompanies the white matter injury in infants and children that suffer from diffuse PVL (Silbereis et al., 2010).

Treatment options are clearly urgently required to prevent the brain damage and associated memory deficits that follow extremely premature birth (Volpe et al., 2011). In this regard, a valid animal model is required. Current small-animal (e.g., rat) models of preterm brain injury typically fail to generate a spectrum of pathology and behavior that closely resembles that ob- 
served in humans, although they do provide important initial answers to numerous cellular, molecular, and therapeutic questions (Silbereis et al., 2010). Most small-animal models also fail to replicate repeated hypoxia during the equivalent time period immediately after extremely preterm birth (Oorschot et al., 2007; Scafidi et al., 2009). There is also a paucity of long-term neuropathological data and behavioral testing in the currently available animal models of hypoxic brain injury (Scafidi et al., 2009).

In this study, we tested the hypothesis that exposure of male rats to repeated hypoxia from postnatal day 1 (P1) to P3 induces the characteristic spectrum of short- and long-term white matter neuropathological injury, gray matter volume loss, and longterm memory deficits seen in children born extremely prematurely. P1-P3 in the rat is widely believed to be the developmental equivalent of the second-trimester human brain at 22-28 weeks of gestation, particularly in terms of white matter development (Volpe et al., 2011). Specifically, we investigated whether repeated hypoxic male rats had a loss of (1) pre-oligodendrocytes at P4, (2) cerebral white and gray matter volume and of myelin, with no cortical or striatal neuronal cell loss, at P14/P15, and (3) cerebral myelin and memory deficits in adulthood.

\section{Materials and Methods}

Animals. All animal procedures were approved by the Committee on Ethics in the Care and Use of Laboratory Animals at the University of Otago (Dunedin, New Zealand). Seventy-eight male Sprague Dawley pups from 13 different litters were used. The day of birth of the pups was defined as P0. Dams, pups, and weaned animals were housed in a $12 \mathrm{~h}$ light/dark cycle in a temperature- and humidity-controlled room. Food and water were available ad libitum, except during behavioral experiments. Pups were weighed daily from P1 to P35 and twice weekly thereafter. The pups used for the quantitation of the absolute number of pre-oligodendrocytes at $\mathrm{P} 4$ and the myelin surface area at P14 (see below) were littermates from three different litters. The pups used for the quantitation of the axon/myelin $g$-ratio and the absolute number of mature oligodendrocytes (see below) were littermates from another three different litters.

Repeated hypoxia or repeated normoxia. Exposure to repeated hypoxia or repeated normoxia was on $\mathrm{P} 1-\mathrm{P} 3$ as described previously (Oorschot et al., 2007). Briefly, the repeated hypoxic pups were exposed to humidified $1.5 \%$ oxygen, $5 \%$ carbon dioxide, and $93.5 \%$ nitrogen at $37^{\circ} \mathrm{C}$ every $2 \mathrm{~h}$, for $12.25 \mathrm{~h} /$ day from P1 to P3. The rationale for the inclusion of carbon dioxide has been discussed previously (Oorschot et al., 2007). During $\mathrm{P} 1-\mathrm{P} 3$, the first exposure commenced at 7:00 A.M. and the last exposure commenced at 7:00 P.M., yielding a total of seven exposures per day. Each hypoxic exposure lasted 15 min on P1 and 14 min on P2 and P3. This protocol induced apnea (i.e., a respiratory pause of $>15 \mathrm{~s}$ ) and was the maximum survivable insult as determined from pilot experiments. Repeated normoxic pups underwent the same experimental paradigm, but the exposure was to humidified normal air at $21 \%$ oxygen with a balance of nitrogen. After each exposure, all pups in a litter were returned to the dam to allow feeding.

Tissue processing and serial sectioning. Immunohistochemical/stereological experiments were undertaken to measure O4-positive preoligodendrocytes at P4, glutathione $S$-transferase- $\pi$ (GST- $\pi$ )-positive mature oligodendrocytes at P13/P14, and myelin at P14 and P545. Each animal was anesthetized with ketamine/xylazine $(67$ and $6.7 \mathrm{mg} / \mathrm{kg}$, i.p., respectively) and intracardially perfused with $4 \%$ paraformaldehyde in $0.1 \mathrm{M}$ phosphate buffer, $\mathrm{pH}$ 7.2. Each brain was cryoprotected in $30 \%$ sucrose in $0.1 \mathrm{M}$ phosphate buffer, $\mathrm{pH} 7.2$, and frozen at $-80^{\circ} \mathrm{C}$. For the O4 experiment, the entire right cerebral hemisphere from eight rats was coronally sectioned in a cryostat into $50 \mu \mathrm{m}$ serial sections. After a random start, every eighth section was systematically sampled throughout the callosal white matter that is enriched with O4-positive oligodendroglia (Back et al., 2002). For the GST- $\pi$ experiment, the entire left cerebral hemisphere from eight rats (two pairs at P13 and two pairs at P14) was coronally sectioned in a cryostat into $5 \mu \mathrm{m}$ serial sections. After a random start, every 100th section pair was systematically sampled throughout the callosal white matter from the start of the caudate-putamen to the end of the hippocampus. Each systematically sampled section pair was collected onto a 3-aminopropylthriethoxy-silane-coated slide. For the myelin experiments, the left cerebral hemisphere from 16 P14 rats and 19 P545 rats was sagittally sectioned in a cryostat into $50 \mu \mathrm{m}$ serial sections. After a random start, every fourth section (P14) or every 12th section (P545) was systematically sampled throughout the cerebral white matter. These sampled sections were then processed for immunohistochemistry and analyzed stereologically (see below).

Histological/stereological experiments were completed at P14 to measure the volume of brain substructures and absolute neuronal number. Each animal was anesthetized with ketamine/xylazine $(67$ and $6.7 \mathrm{mg} / \mathrm{kg}$, i.p., respectively) and intracardially perfused with $4 \%$ paraformaldehyde $/ 0.1 \%$ glutaraldehyde in $0.1 \mathrm{~m}$ phosphate buffer, $\mathrm{pH}$ 7.2. The right cerebrum from $17 \mathrm{P} 14$ rats was postfixed in Formalin for 5 d, dehydrated, embedded in Technovit, serially sectioned into $40 \mu \mathrm{m}$ sections, and stained as described previously (Oorschot, 1996).

Electron microscopic/stereological experiments were completed at P14/P15 to measure the $g$-ratio and the number and diameter of myelinated axons in the callosal periventricular white matter. Each animal was anesthetized with ketamine/xylazine $(67$ and $6.7 \mathrm{mg} / \mathrm{kg}$, i.p., respectively) and intracardially perfused with $2 \%$ paraformaldehyde $/ 2.5 \%$ glutaraldehyde in $0.1 \mathrm{~m}$ phosphate buffer, $\mathrm{pH} 7.2$. The left cerebrum from 10 $\mathrm{P} 14 / \mathrm{P} 15$ rats (one pair at $\mathrm{P} 14$ and four pairs at $\mathrm{P} 15$ ) was postfixed in fresh fixative for $5-6 \mathrm{~d}$ at $4^{\circ} \mathrm{C}$. Each cerebral hemisphere was cut in the sagittal plane, from lateral to medial, into $17-20$ serial $200 \mu \mathrm{m}$ vibratome sections and then serial $50 \mu \mathrm{m}$ vibratome sections thereafter. Using Plates $168-170$ by Paxinos and Watson (2007) and a Seiwa dissecting microscope, medial $50 \mu \mathrm{m}$ sections were viewed to identify the anterior limb of the anterior commissure in the cerebrum and its continuity with the intrabulbar anterior commissure in the medially located olfactory bulb. The continuity between these structures becomes discontinuous medially (Paxinos and Watson, 2007, their Plate 168) and laterally. The first section that contained the medial discontinuity was selected for processing for transmission electron microscopy. This ensured that the same location in the brain was investigated for each animal (Tos et al., 2008). Each selected section also contained the callosal white matter surrounding the lateral ventricle (Paxinos and Watson, 2007, their Plate 168). This callosal periventricular white matter was the specific biological region of interest for measuring the $g$-ratio and the diameter and number of myelinated axons.

Each selected section per rat was osmicated, dehydrated, embedded in resin, and prepared for ultramicrotomy as described previously (Partadiredja et al., 2003), with the exception that EMbed 812 resin was used and the resin-infiltrated sections were flat-embedded between silanized slides. The EMbed 812 resin consisted of $44.25 \%$ EMbed 812 resin, $35.4 \%$ dodecenyl succinic anhydride, $17.7 \%$ methyl nadic anhydride, and $2.65 \%$ benzyldimethylamine. Before ultramicrotomy, the callosal periventricular white matter in each flat-embedded section was trimmed to a trapezoid shape (Partadiredja et al., 2003, their Fig. 1, "parietal") to yield the final resin block for each animal. Ultrathin sections from each block were cut at $80 \mathrm{~nm}$, collected onto Formvar-coated grids, and stained with uranyl acetate and lead citrate in a 2168 Carlsberg Ultrostainer (LKB Bromma). The sections were then viewed in a Philips CM100 transmission electron microscope.

Immunohistochemistry. Free-floating sections in tissue culture wells and sections affixed to slides (for GST- $\pi$ staining) were incubated in 5\% heat-inactivated goat serum (for O4 and GST- $\pi$ staining) or donkey serum (for myelin staining; Sigma-Aldrich) in PBS for $1 \mathrm{~h}$ to block nonspecific binding sites. For O4 staining, sections were then incubated in a monoclonal mouse anti-O4 primary antibody (1:100; Millipore Bioscience Research Reagents) in antibody diluting buffer ( $1 \%$ bovine serum albumin; A-2153; (Sigma) in $0.1 \mathrm{M}$ PBS overnight at $4^{\circ} \mathrm{C}$. To stain myelinated axons, sections were incubated in a monoclonal mouse antibody to myelin basic protein (1:200; Millipore Bioscience Research Reagents). Sections were then incubated in a biotinylated anti-mouse secondary antibody (1:200; Vector Laboratories) in antibody diluting buffer with $5 \%$ goat or donkey serum, respectively, for $1 \mathrm{~h}$. To stain mature oligo- 
dendrocytes, sections were incubated in a polyclonal rabbit antibody to GST- $\pi$ (1:500; MBL). Sections were then incubated in a biotinylated anti-rabbit secondary antibody (1:200; Vector Laboratories) in antibody diluting buffer with $5 \%$ goat serum for $1 \mathrm{~h}$. After washing, sections were incubated in $0.30 \%$ hydrogen peroxidase in PBS for $10 \mathrm{~min}$ to inactivate the endogenous peroxidases and washed again. All sections were then incubated in a tertiary antibody, streptavidin-conjugated horseradish peroxidase (1:200; Vector Laboratories), for $1 \mathrm{~h}$. The antigen/antibody complex in the sections was visualized with 3-amino-9-ethyl-carbazole (Sigma) in acetate buffer. The sections were then mounted onto gelatincoated slides and coverslipped in glycerol gelatin (Sigma) for subsequent stereological analyses. Negative control sections with the primary antibody omitted were included in all experiments to confirm that the staining was specific to the O4 cells, GST- $\pi$ cells, or myelin.

Stereology: general methods. All sections were coded before stereologi$\mathrm{cal}$ analyses. To measure the numerical density $\left(N_{v}\right)$ of O4-positive cells and neurons, the surface density $\left(S_{v}\right)$ of myelin, and neuronal nuclear volume, each sampled subvolume in each sampled section was viewed using a $100 \times$ oil-immersion objective lens (numerical aperture 1.4) on an Olympus BH-2 light microscope. The microscope was also fitted with an automated mechanical stage, an electronic microcator (to measure height in the $z$-plane), and a video camera that projected the image onto an adjacent television monitor. Overlaying the television monitor was an unbiased sampling frame, either $80 \times 80 \mathrm{~mm}\left(\right.$ for $\left.N_{v}\right)$ or $140 \times 70 \mathrm{~mm}$ (for $S_{v}$ ), with two inclusion lines and two exclusion lines (Gundersen, 1977). With the sampling frame and the automated stage, the entire brain region of interest (e.g., the callosal white matter) in every selected section was sampled using a random start and systematic sampling (e.g., every 16th subvolume) thereafter. If a cell of interest (e.g., an O4-positive cell) fell on or inside the inclusion lines of an unbiased sampling frame, it was counted. However, if it fell on the exclusion lines, it was not. To measure the $N_{v}$ of GST- $\pi$-positive mature oligodendrocytes, these generic sampling principles were applied to another counting method detailed below. At the completion of stereological analyses, data were decoded, and the average of the parameter of interest was calculated for the repeated normoxic and repeated hypoxic groups.

Absolute number of O4-positive pre-oligodendrocytes in the callosal white matter at P4. The parasagittal corpus callosum, the supracallosal region (i.e., cingulum; Paxinos and Watson, 1998), and the lateral corpus callosum/white matter are the areas most enriched with O4-positive oligodendroglia (Back et al., 2002). The boundaries of this callosal white matter were reproducibly defined using extrinsic landmarks (Fig. 1). The absolute number of O4-positive cells in the callosal white matter was measured using the optical fractionator method (Gundersen et al., 1988). O4-positive cells were identified by a specific halo of immunostaining in the cell membrane (Fig. 2A). Counting was conducted at $3400 \times$ and started at a depth of $5 \mu \mathrm{m}$ into each section and continued for $10 \mu \mathrm{m}$ to a depth of $15 \mu \mathrm{m}$. If an O4-postive cell was fully in focus at $5 \mu \mathrm{m}$, it was counted, and all focused O4-positive cells that were observed through to $14 \mu \mathrm{m}$ were counted. A cell that was in focus between 5 and $14 \mu \mathrm{m}$ in the $z$-plane was only counted if it was out of focus at $15 \mu \mathrm{m}$. The total thickness of the section was also measured at each area sampled to yield an accurate measure of section thickness. The absolute number $(N)$ of O4-positive cells in the callosal white matter was calculated from $N=$ $\left(\Sigma Q^{-}\right) \times($ssf $) \times($asf $) \times(s)$, where $\Sigma Q^{-}$is the sum of all O4-positive cells counted in the subvolumes of the callosal white matter, ssf is the section sampling fraction [the average inverse of the height of the subvolume $(h)$ of $10 \mu \mathrm{m}$ divided by the thickness $(t)$ of each sampled section], asf is the area sampling fraction (the inverse of the fraction of the total area sampled), and $s$ is the interval between the sampled sections. The value for asf was always 16 , and $s$ was always $8 . \Sigma Q^{-}$and ssf varied for each rat.

Absolute surface area of cerebral myelin at P14 and P545. Specific structures included in this analysis were the subcortical white matter (Fig. 2I), fornix, corpus callosum, internal capsule (Fig. 2C,D), globus pallidus, lateral olfactory tract, optic tract (Fig. 2C,D), and the anterior commissure. These structures were identified using the borders of the immunostaining for myelin basic protein and Plates $79 a-89$ by Paxinos and Watson (1998). The $S_{v}$ of myelin in a known subvolume of white matter was measured using the method of Baddeley et al. (1986). An optical disector sampling method was used at $6800 \times$. A transparent acetate sheet, with four cycloid arcs (Baddeley et al., 1986) that were used for intercept points and four reference points, was placed over the image of the section (Fig. $2 E, I$ ) and fixed to the monitor. After a random start, every 24 th subvolume was sampled. When at a sampled subvolume, a random focal depth within the subvolume was chosen for counting. The number of arcs that intercepted the myelinated axons were counted, as well as the number of reference points that were in the white matter. An example of the myelinated axon tracts is seen in Figure 2I. After all the sections from a hemisphere were counted, the data were summed to obtain a final count of the number of myelin intercepts $\left(\sum I\right)$ and reference points $\left(\sum P\right)$ within each hemisphere. $S_{v}$ was then calculated from $S_{v}=2 \times(p / I) \times M \times\left(\sum I / \sum P\right)$, where $p$ is the number of reference points in the white matter, $I$ is the length of the cycloid $\operatorname{arc}(1.52 \mathrm{~cm}), p / I$ is the ratio of points to cycloid length (1/1.52), $M$ is magnification, $\sum I$ is the total number of intersects with myelin, and $\sum P$ is the total number of reference points in the white matter (Baddeley et al., 1986). A microcator also allowed for section thickness ( $t$; see below) to be measured from the upper surface of the section to the lower surface. Two measures of tissue thickness were taken from different locations in each section, for both white matter and gray matter, and averaged to obtain their respective thicknesses for each section.

The total surface area of cerebral myelin, $S_{a b}$, in the left cerebral hemisphere was determined from $S_{a b}=S_{v} \times \operatorname{Vref}(\mathrm{WM})$, where Vref (WM) was the total reference volume of the white matter. Vref (WM) was measured using Cavalieri's method (Michel and Cruz-Orive, 1988; Gundersen et al., 1999). Briefly, each sampled section was projected onto a white screen at a known magnification, and a lattice of regularly arranged points with an interpoint distance of $3 \mathrm{~cm}$ was superimposed randomly over it. The number of points $(P)$ falling on the specific myelin-immunostained structures of interest were counted for each section and summed $\left(\sum P\right)$ for the entire hemisphere. Vref (WM) was then determined from $\sum P \times a(p) \times t \times s$, where $a(p)$ is the real area that each point represents, $t$ is the section thickness of the white matter (obtained using the optical disector method explained above), and $s$ is the section interval of 4 (P14) or 12 (P545).

A tissue shrinkage correction was applied to correct for the effect of shrinkage during tissue processing (Oorschot, 1994). The formula used was as follows: Volume shrinkage $=$ volume before processing/volume after processing. The volume before processing was the weight of the hemisphere directly after it was dissected from the cranium (converted into cubic millimeters to yield a volume). The volume after processing was the Vref of the hemisphere. To measure the Vref of the entire left cerebral hemisphere, each sampled section was projected onto a white screen at a known magnification, and a lattice of regularly arranged points with an interpoint distance of $2 \mathrm{~cm}$ was superimposed randomly over it. The number of points falling on each entire section were then counted and summed across all sections to yield a total sum of points, $\sum P$, for the entire hemisphere. The same Vref formula outlined above was then used to calculate the Vref of the hemisphere. However, an average of the tissue thickness from both the gray matter and the white matter was used. The final $S_{a b}$ of myelin in the left hemisphere was then determined from $S_{a b}$ (uncorrected) $\times$ volume shrinkage factor (Baddeley et al., 1986).

Axon/myelin g-ratio and number of myelinated axons in the callosal periventricular white matter at P14/P15. A deficit in myelin, if detected in repeated hypoxic rats, may reflect a decreased amount of myelin per myelinated axon (i.e., an increased $g$-ratio) and/or a decrease in the number of myelinated axons. The $g$-ratio and number of myelinated axons per $100 \mu \mathrm{m}^{2}$ were measured in $6-10$ coded digital electron micrographs, chosen systematically after a random start, throughout an $80 \mathrm{~nm}$ section of identified callosal periventricular white matter per rat (see above). Digital electron micrographs were photographed at $9700 \times$ in a Philips CM100 transmission electron microscope. Using Fiji software, an unbiased sampling frame, $8500 \mathrm{~nm}$ width $\times 6500 \mathrm{~nm}$ height, was applied to each digital electron micrograph to count the number of myelinated axons that fell within its inclusion lines (Fig. $3 A, B$ ). The axons sampled for number per $100 \mu \mathrm{m}^{2}$ were also measured for their $g$-ratio. To obtain 
A

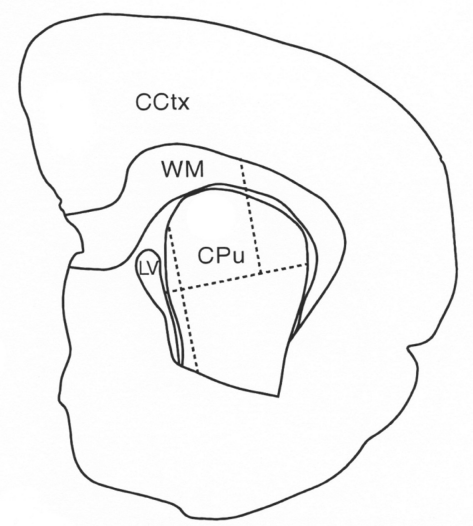

B

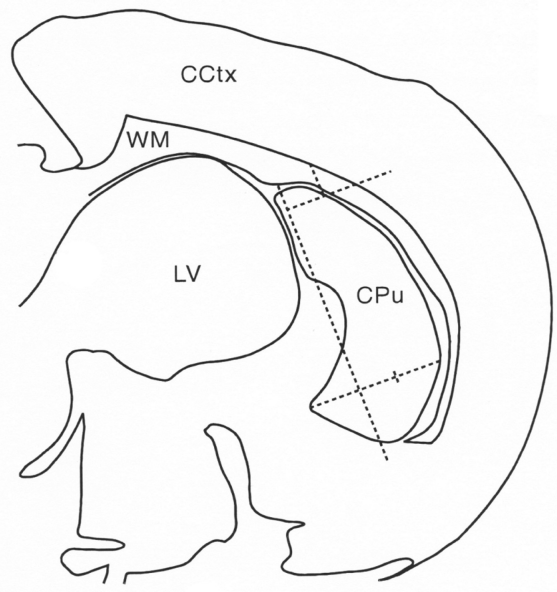

C

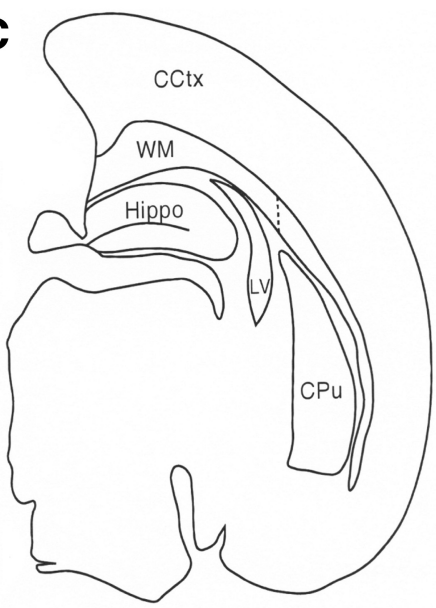

D

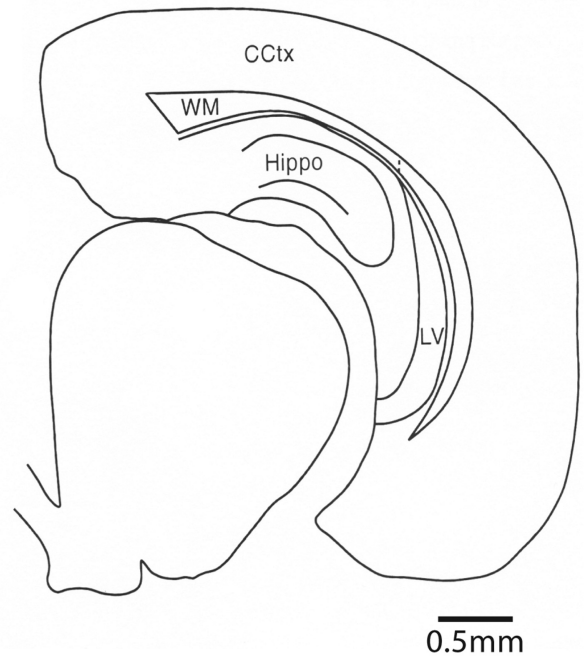

Figure 1. Illustration of the boundaries used to define the callosal white matter. $\boldsymbol{A}-\boldsymbol{D}$ are illustrations of coronal sections through a $\mathrm{P} 4$ rat brain proceeding from anterior $(\boldsymbol{A})$ to posterior $(\boldsymbol{D})$. The respective section numbers are $49(\boldsymbol{A}), 73(\boldsymbol{B}), 89(\boldsymbol{C})$, and $113(\boldsymbol{D})$, with all sections derived from the same rat. In all sections, the medial border of the callosal white matter (WM) was defined as the point at which the corpus callosum meets the edge of the section. In the sections anterior to the hippocampus (i.e., $\boldsymbol{A}, \boldsymbol{B}$ ), the lateral border of the callosal white matter was delineated by first measuring the maximum horizontal width of the striatum [i.e., caudate-putamen (CPu)] in each section. The vertical axis for this horizontal measurement was usually the vertical plane of the entire medial boundary of the caudate-putamen and its adjacent lateral ventricle (LV; see $A)$. When the lateral ventricle abutted on less than one-third of the medial surface of the caudate-putamen, the superomedial boundary of the caudate-putamen was used as the vertical axis for this horizontal measurement $(\boldsymbol{B})$. The horizontal measurement was completed on a carefully drawn camera lucida diagram of all the key brain regions in each section $(\boldsymbol{A}, \boldsymbol{B})$. Each section was carefully drawn by projecting it onto a whiteboard at $50 \times$. The maximum horizontal width of the caudate-putamen was then divided by three to yield one-third of the maximum distance. This one-third distance was marked on the diagram from the most lateral edge of the caudate-putamen. A vertical line from this lateral one-third mark was then drawn superiorly through the white matter, in a plane that was parallel to the vertical line of the medial boundary of the caudate-putamen, to demarcate the lateral boundary of the callosal white matter $(\boldsymbol{A}, \boldsymbol{B})$. In the more posterior sections that contained the hippocampus, the lateral border was defined by first finding the most superior point of the caudate-putamen. A vertical line was then drawn superiorly from this point through the white matter, in a plane that was parallel to the vertical medial boundary of the caudate-putamen $(\boldsymbol{C})$, to demarcate the lateral boundary of the callosal white matter. When the caudate-putamen was no longer present in the most posterior sections, the lateral border was defined by first finding the most superior point of the lateral ventricle. A vertical line was then drawn superiorly from this point through the white matter, in a plane that was parallel to the vertical medial boundary of the section (D). The white matter below the exclusion line was not sampled, whereas the white matter from above the line around to the medial edge of the corpus callosum was sampled. CCtx, Cerebral cortex; Hippo, hippocampus.

the $g$-ratio of each sampled myelinated axon, the diameter of the axon $(D)$, the diameter of adjacent oligodendrocytic cytoplasm $(O)$ (if present), and the diameter of the myelin sheath $(M)$ was measured using Fiji software at $19,400 \times$ (Fig. $3 C, D)$. The $g$-ratio for an axon was determined from $D /((M-O)+D)$. The measurement of axon diameter was also used independently of the $g$-ratio measure to secondarily investigate whether repeated hypoxia altered axonal caliber. The rules and definitions for measuring the number and diameter of myelinated axons were as defined previously (Partadiredja et al., 2003; Fig. 3A-D). Briefly, the diameter of an axon was defined as "the longest profile diameter perpendicular to the longest axis of an axon." The average $g$-ratio, number of myelinated axons per $100 \mu \mathrm{m}^{2}$, and the average diameter of myelinated axons were calculated for each rat. The latter two measures were then corrected for processing-induced area and linear shrinkage, respectively, as described previously (Partadiredja et al., 2003). Data were then decoded.

Absolute number of GST- $\pi$-positive mature oligodendrocytes in the callosal white matter at P13/P14. Abnormalities in myelin expression without an overt loss of mature oligodendrocytes are observed clinically in extreme prematurity (Billiards et al., 2008). The absolute number of GST- $\pi$-positive mature oligodendrocytes in the callosal white matter was measured using Cavalieri's method to estimate the Vref and the physical disector method (Gundersen et al., 1988; Oorschot, 1994) to measure the $N_{v}$ of oligodendrocytes in pairs of adjacent $5 \mu \mathrm{m}$ sections. The optical disector method was unable to be performed because of minimal penetration of antibodies through thicker $50 \mu \mathrm{m}$ sections of the P13/P14 brain.

To measure the Vref, the medial boundary of the callosal white matter was defined as indicated in Figure 1. For the lateral boundary in sections 
A

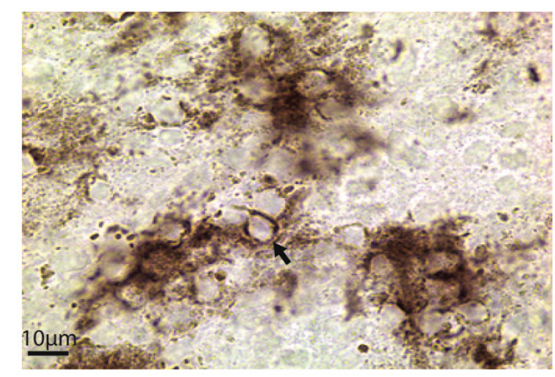

B
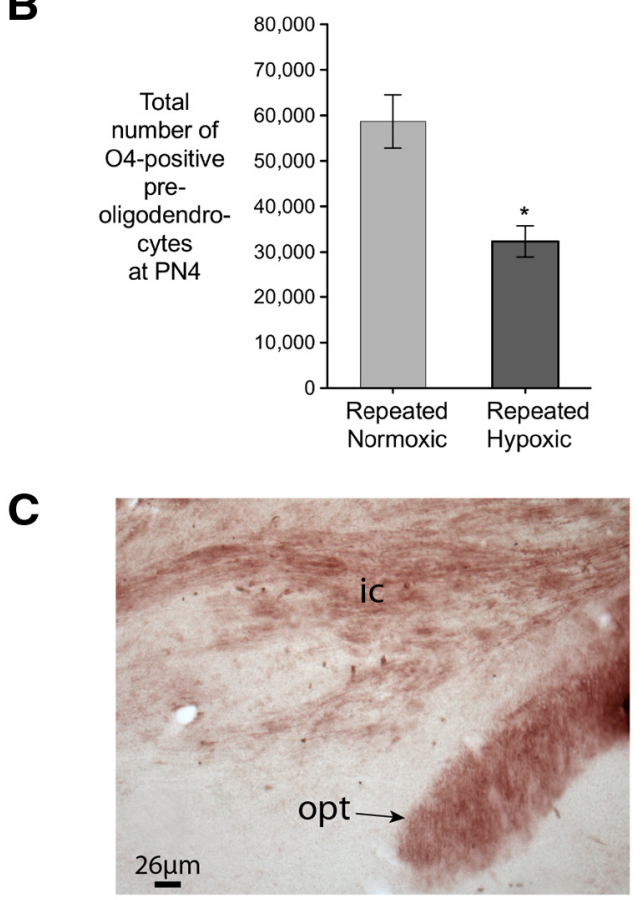

D

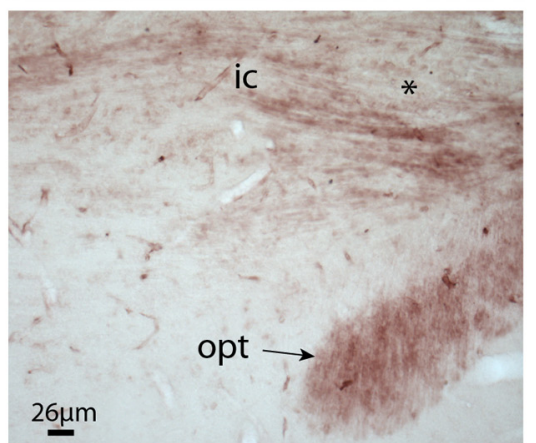

$\mathbf{E}$

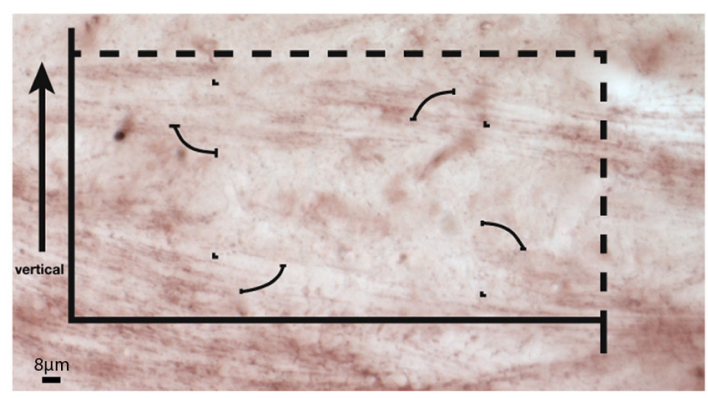

$\mathbf{F}$

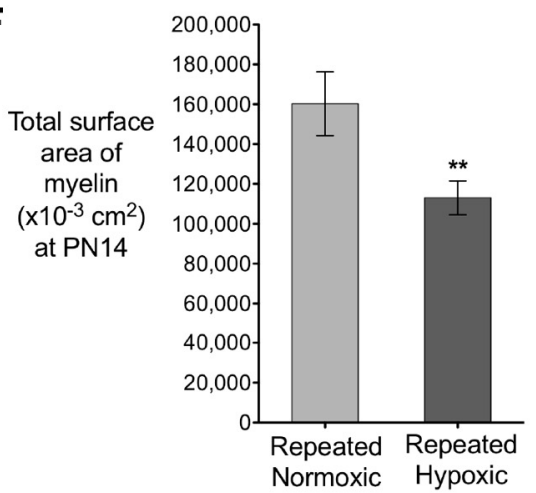

G
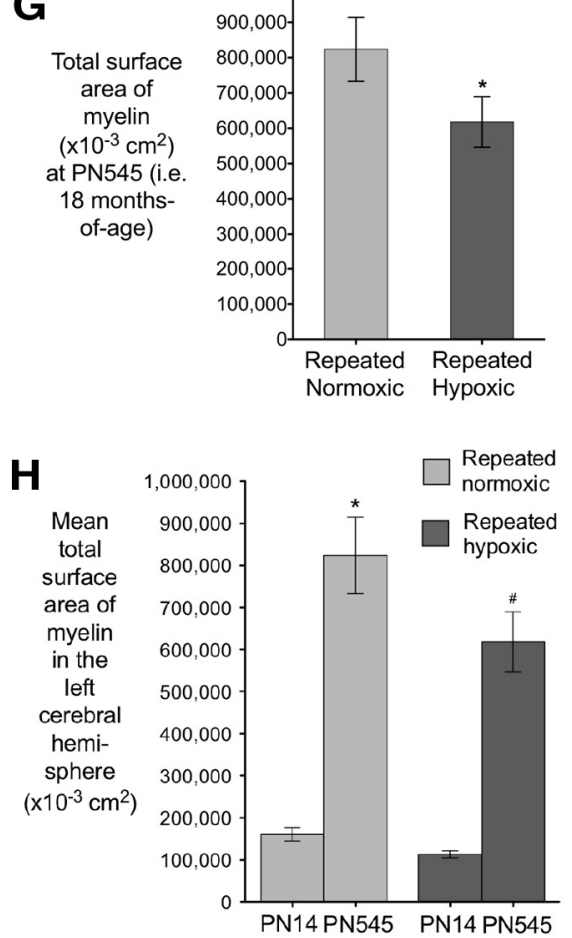

I

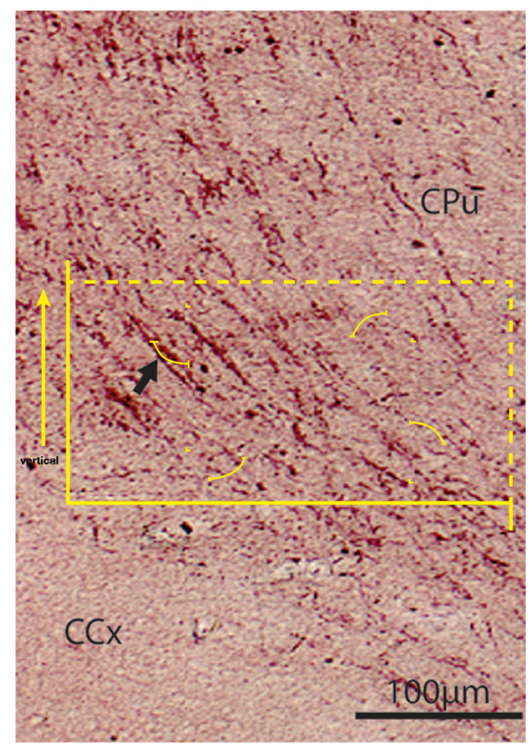

Figure 2. Rats exposed to repeated hypoxia from P1 to P3 had a significant loss of 04-positive pre-oligodendrocytes at P4, reduced cerebral myelin at P14 and P545 (i.e., 18 months of age), and a similar increase in myelin between P14 and P545 when compared with rats exposed to repeated normoxia from P1 to P3. A, Immunohistochemically identified (Figure legend continues.) 
that contained the caudate-putamen, first a line was drawn parallel to the medial boundary of the brain at the level of the cingulate cerebral cortex. The lateral boundary of the callosal white matter was then defined by drawing another line within the brain, parallel to the medial line, at the most lateral junction between the lateral wall of the lateral ventricle and the medial surface of the caudate-putamen. For more posterior sections that did not contain the caudate-putamen but contained the hippocampus, a medial line was drawn as indicated above. A lateral parallel line was then drawn through the brain at the most lateral boundary of the thalamus or midbrain to demarcate the lateral boundary of the callosal white matter. The superior and inferior boundaries of the callosal white matter were then defined using the borders of immunostaining for GST- $\pi$ (Fig. $4 A, B)$. To measure the Vref of the entire left callosal white matter, a lattice of regularly arranged points with an interpoint distance of 0.15 $\mathrm{mm}$ was superimposed randomly over each sampled reference section at $90 \times$ using Stereoinvestigator software. The number of points falling on the callosal white matter were then counted and summed across all sections to yield a total sum of points, $\sum P$, for the entire hemisphere. The same Vref formula outlined above was then used to calculate the Vref of the left callosal white matter for each animal, with $t=0.005$ $\mathrm{mm}$ and $s=100$.

To measure the $N_{v}$ of GST- $\pi$-positive mature oligodendrocytes, each sampled subvolume in each sampled section pair was viewed using a $40 \times$ objective lens on an Olympus BX-51 light microscope. The microscope was fitted with a video camera that projected the image onto an adjacent computer screen. Using Stereoinvestigator software and an automated stage, the entire callosal white matter of interest in every reference section of a selected pair was sampled using a random start and systematic sampling of every 20th subvolume thereafter. Each sampled volume in the reference, and adjacent look-up, section, was digitally photographed. Digital images of the paired sampled subvolumes were analyzed at $1480 \times$ on another computer using Photoshop CS5 software and an unbiased sampling frame of $95 \times 95 \mathrm{~mm}$, with cell inclusion and exclusion criteria as defined above.

GST- $\pi$-positive mature oligodendrocytes within the callosal white matter were identified by specific immunostaining in their somal cytoplasm and processes (Fig. $4 C, D$ ). If the nucleus of a GST- $\pi$-positive oligodendrocyte was in the reference section (Fig. $4 A, C$ ) but not in the adjacent look-up section (Fig. $4 B, D$ ), the cell was counted as a disector

\section{$\leftarrow$}

(Figure legend continued.) 04-positive pre-oligodendrocyte (arrow) in the callosal white matter at P4. Because the 04-positive cells are located in a $50-\mu \mathrm{m}$-thick section, other cells are in focus at a different depth to the arrowed cell. Note also that this image was taken at $1000 \times$, whereas in this study each 04-positive cell was identified at $3400 \times$ and counted as it clearly came into focus (see Materials and Methods). $\boldsymbol{B}$, Total (i.e., absolute) number of 04-positive pre-oligodendrocytes in the callosal white matter at P4. Two groups of rats were used: (1) repeated normoxic $(n=4)$ and (2) repeated hypoxic $(n=4)$ (paired two-tailed Student's t test, $\left.{ }^{*} p=0.032\right) . C, D, E, I$, Sagittal sections stained immunohistochemically with myelin basic protein for a P14 repeated normoxic rat (C), P14 repeated hypoxic rat $(\boldsymbol{D}, \boldsymbol{E})$, and P545 repeated normoxic rat $(\boldsymbol{D})$. The area indicated with an ${ }^{*}$ in $\boldsymbol{D}$ is illustrated at higher magnification in $\boldsymbol{E}$. Note the staining in the internal capsule (ic) and optic tract (opt) is paler in the repeated hypoxic animal (compare $\boldsymbol{D}$ with $\boldsymbol{C}$ ). The sections in $\mathbf{C}$ and $\boldsymbol{D}$ were immunostained at the same time, photographed at the same lighting intensity, and have the same section thickness. This qualitative difference reflects the quantitatively lower absolute lower surface area of cerebral myelin in the repeated hypoxic group versus the repeated normoxic group at P14 (F). Cycloid arcs intersecting myelinated axons to measure the $S_{V}$ using an unbiased sampling frame are illustrated in $\boldsymbol{E}$ at $P 14(395 \times)$ and $/$ at $P 545(330 \times)$. Note that the actual quantification of the $S_{V}$ of myelin was completed at a magnification of $6800 \times$. The stronger staining in $/$ reflects the amount of myelin in a P545 brain. In $I$, a myelin basic protein-positive axon is indicated in the subcortical white matter (arrow) between the cerebral cortex ( $(\mathrm{CX})$ and caudate-putamen ( $\mathrm{CPu}$; striatum). $\boldsymbol{F}$, Absolute (i.e., total) surface area of cerebral myelin at P14. Two groups of rats were used: (1) repeated normoxic $(n=8)$ and (2) repeated hypoxic $(n=8)$ (two-tailed Student'st test, $\left.{ }^{* *} p=0.021\right)$. G, Absolute (i.e., total) surface area of cerebral myelin at P545 (i.e., 18 months of age). Two groups of rats were used: (1) repeated normoxic $(n=9)$ and (2) repeated hypoxic $(n=10)$ (one-tailed Student's $t$ test, ${ }^{*} p=$ 0.045). $\boldsymbol{H}$, Absolute (i.e., total) surface area of myelin at P14 and P545 (i.e., 18 months of age). Same rats as $F$ and $\boldsymbol{G}$ (two-tailed Student's t test, ${ }^{*} p<0.0001$, repeated hypoxic rats at $P 14$ compared with P545; $" p<0.0001$, repeated normoxic rats at P14 compared with P545). Reliable estimates were obtained for all stereological data in this figure (see also Results). Error bars show SEM. oligodendrocyte for the reference section (Fig. 4C). If the nucleus of a GST- $\pi$-positive oligodendrocyte was in the look-up section but not in the adjacent reference section, the cell was counted as a disector oligodendrocyte for the look-up section (Fig. $4 D$ ). The average number of disector oligodendrocytes per sampled subvolume was calculated from $\Sigma Q^{-}$divided by the total number of sampled subvolumes in the reference and look-up sections, where $\Sigma Q^{-}$is the total number of identified disector oligodendrocytes. Each sampled subvolume equaled the area of the unbiased sampling frame, corrected for magnification, multiplied by the section thickness (i.e., $95 \mathrm{~mm} / 1480 \times 95 \mathrm{~mm} / 1480 \times 0.005 \mathrm{~mm}$; see above). The absolute number ( $N$ ) of GST- $\pi$-positive cells in the callosal white matter was calculated from $N=$ (Vref/volume of an individual sampled subvolume) $\times$ (average number of disector oligodendrocytes per sampled subvolume).

Absolute volume of the cerebral white matter, cerebral cortex, and striatum and the absolute number of neurons in the cerebral cortex and striatum at P14. Cavalieri's method (see above) was also used to measure the absolute volume of the white matter as well as the gray matter of the cerebral cortex and striatum in Technovit sections at P14. Absolute neuronal number in the cerebral cortex and striatum was calculated using the optical disector technique in combination with Cavalieri's method as described previously (Oorschot, 1996; for additional details, see the footnotes for Tables 3 and 4 ).

Measurement of nuclear volume at P14. A deficit in the average nuclear volume of striatal medium-spiny neurons, which comprise $>97 \%$ of neurons in the rat striatum (Oorschot et al., 2002), may contribute to an observed deficit, if detected, in the absolute volume of the striatum in repeated hypoxic rats. The average volume of the neuronal nucleus of striatal spiny projection neurons was measured using the nucleator method (Gundersen et al., 1988). After a random start, every 270th subvolume in the striatum was sampled. A medium-spiny neuron was identified as a neuron that has a large, spherical, or slightly ovoid pale nucleus containing one prominent nucleolus (Oorschot, 1996). Neurons with a slightly darker homogenous nucleus and a nucleolus were also measured (Oorschot, 1996). Sampling of neurons was conducted at $3400 \times$ and started at a depth of $10 \mu \mathrm{m}$ into the section and continued for $15 \mu \mathrm{m}$ to a depth of 25 $\mu \mathrm{m}$. If a neuron was fully in focus at $10 \mu \mathrm{m}$, its nucleus was measured, and all neurons that were observed in focus through to $24 \mu \mathrm{m}$ were measured. A neuron that was in focus between 10 and $24 \mu \mathrm{m}$ in the $z$-plane was only measured if it was out of focus at $25 \mu \mathrm{m}$. Each nucleus was measured at its specific plane of focus between 10 and $24 \mu \mathrm{m}$ into the section.

The nucleolus was used as the point from which measures were taken. The nucleolus was defined as the largest, darkest, centrally located structure in the nucleus (Tandrup, 1993). A sine-weighted test grid for vertical sections was placed on top of an $80 \times 80 \mathrm{~mm}$ unbiased sampling frame (Gundersen et al., 1988). The midpoint of the $1^{3}$ ruler was placed onto the nucleolus, and a random number table was used to choose an angle on which to make the first measurement. The two class levels where the ruler intersected each of the edges of the nucleus were recorded. Another random angle was chosen and the next two class levels were recorded to yield a total of four $1^{3}$ measurements for each neuron. This procedure was followed for all unbiasedly sampled medium-spiny neurons in each disector subvolume. In undertaking these measurements, it was assumed that the neurons had a global isotropic orientation in three-dimensional space. This appears to be the case in both light and electron microscopic observations of these striatal neurons (Oorschot, 1996; Oorschot et al., 2002), yet quantitative evidence is lacking. This assumption was needed because the vertical coronal sections used were not cut in random planes around this vertical axis.

The four $1^{3}$ measurements for each neuron were corrected for magnification. The nucleator formula for the mean nuclear volume of a neuron was then calculated from the following: $\mathrm{V}$ (volume) $=\ln (v)=4 \pi / 3 \cdot \mathrm{av}-$ erage $\left.1^{3}=4 \pi / 3 \cdot\left(1_{1}{ }^{3}+1_{2}{ }^{3}+1_{3}{ }^{3}+1_{4}{ }^{3}\right) / 4\right) \mu \mathrm{m}^{3}$. The average $\pm \mathrm{SD}$ of the nuclear volume for $\geq 100$ measured neurons per rat was then calculated. The data were then decoded and corrected for processing-induced shrinkage. The shrinkage correction factor was calculated as described above (Oorschot, 1994).

Coefficient of error of the stereological estimates. Estimates of precision, termed the coefficient of error $(\mathrm{CE})$, were calculated for the stereological 
A

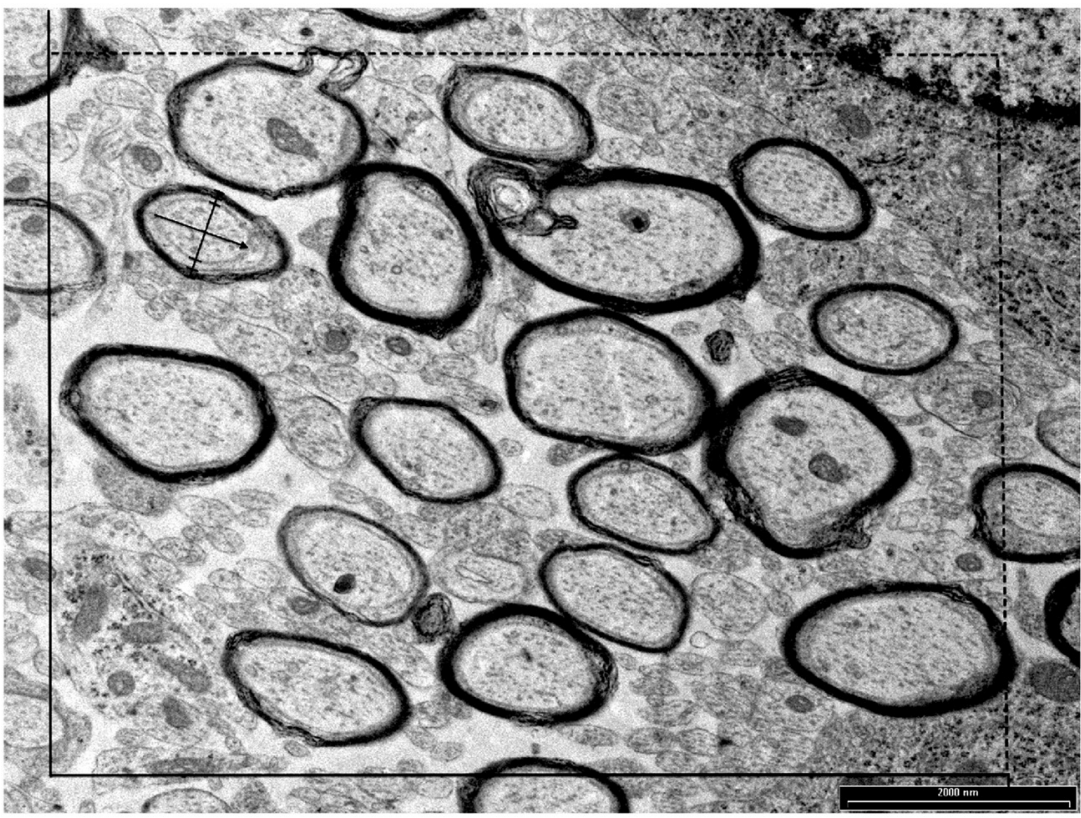

B

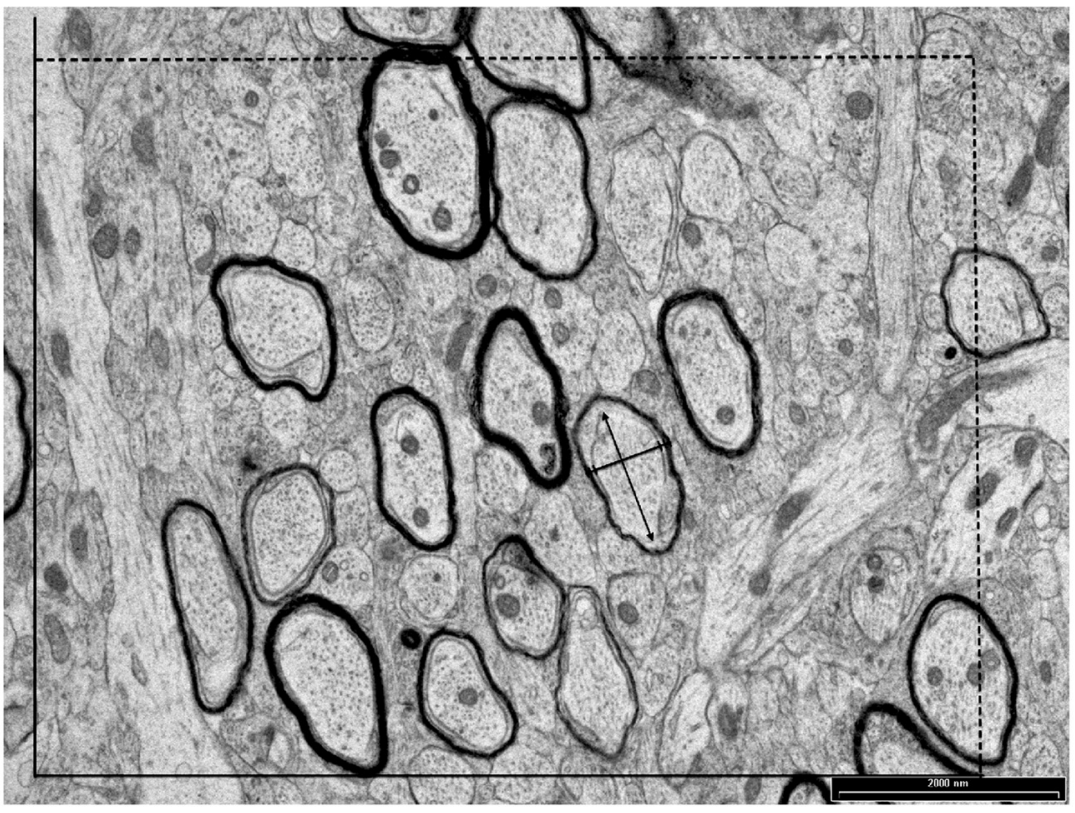

C

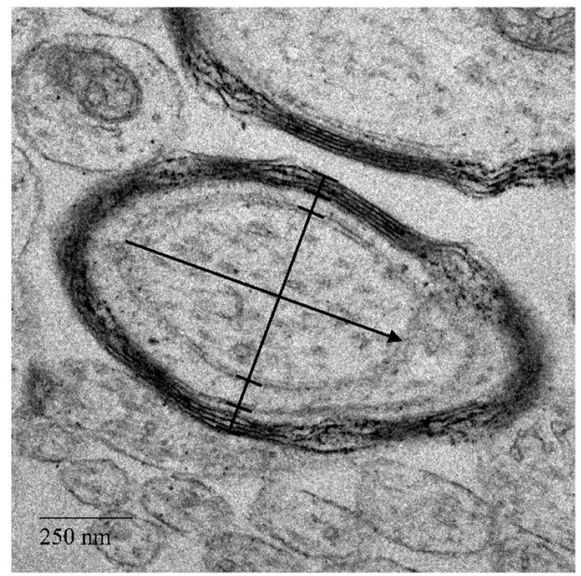

D

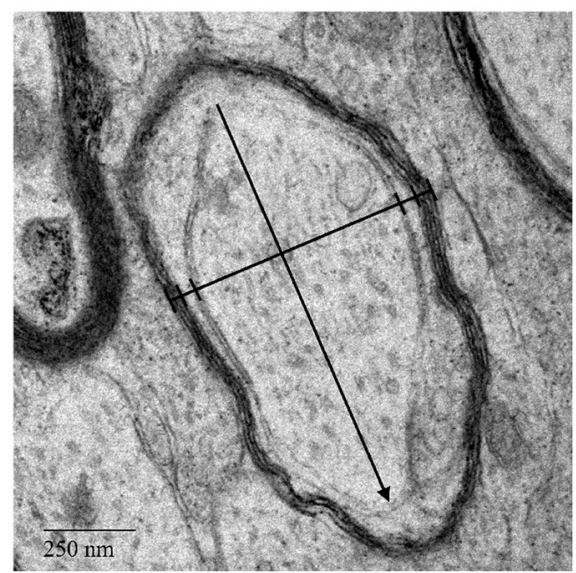

E

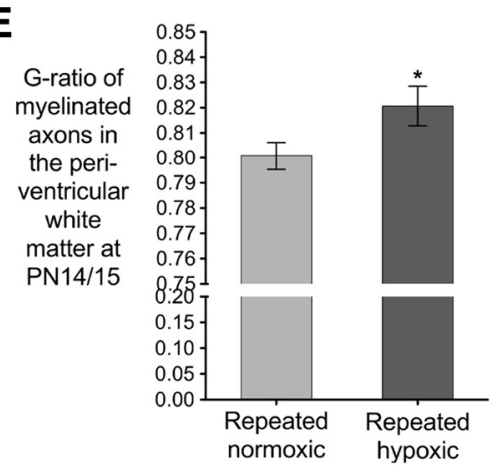

$\mathbf{F}$

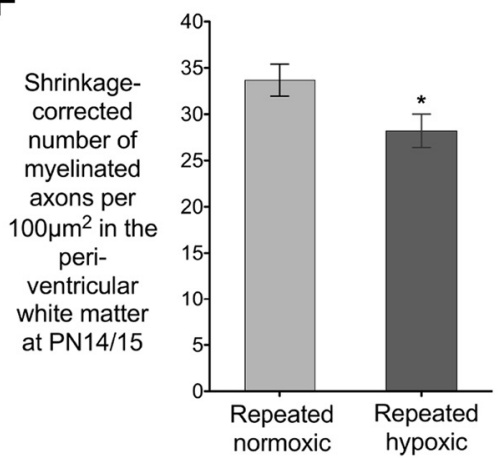

Figure 3. Rats exposed to repeated hypoxia from $\mathrm{P} 1$ to $\mathrm{P} 3$ had significantly thinner myelin per axon (i.e., an increased $g$-ratio) and a significant reduction in the number of myelinated axons per $100 \mu \mathrm{m}^{2}$ in the periventricular white matter at P14/P15. $\boldsymbol{A}-\boldsymbol{D}$, Electron micrographs of a sampled area of the periventricular white matter from a repeated normoxic $(\boldsymbol{A}, \boldsymbol{C})$ or repeated hypoxic $(\boldsymbol{B}$, D) brain. The $g$-ratio and the number of myelinated axons per $100 \mu \mathrm{m}^{2}$ was measured from each sampled area by using the unbiased sampling frame illustrated in (Figure legend continues.) 
A

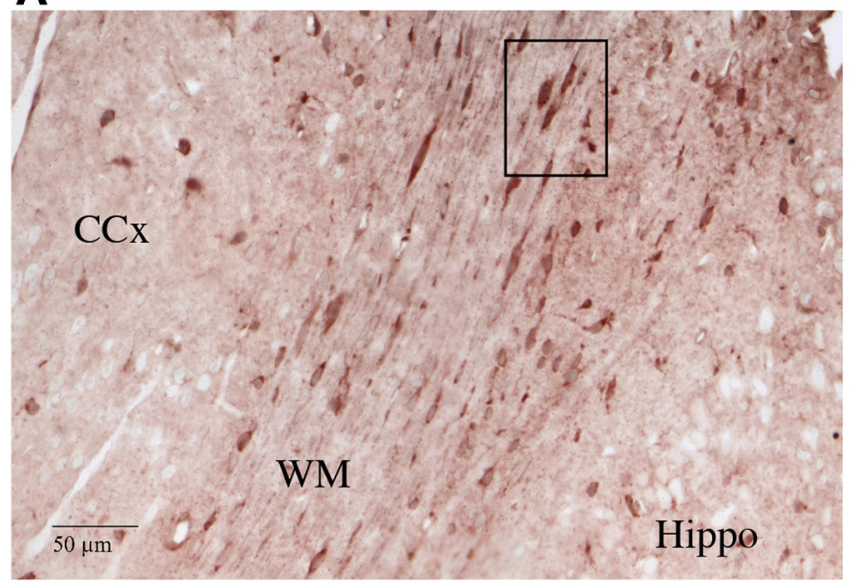

C

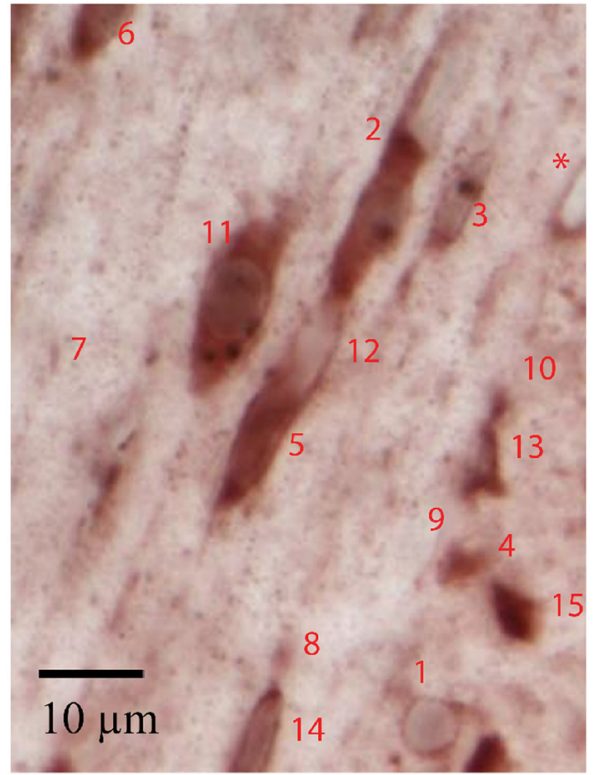

B

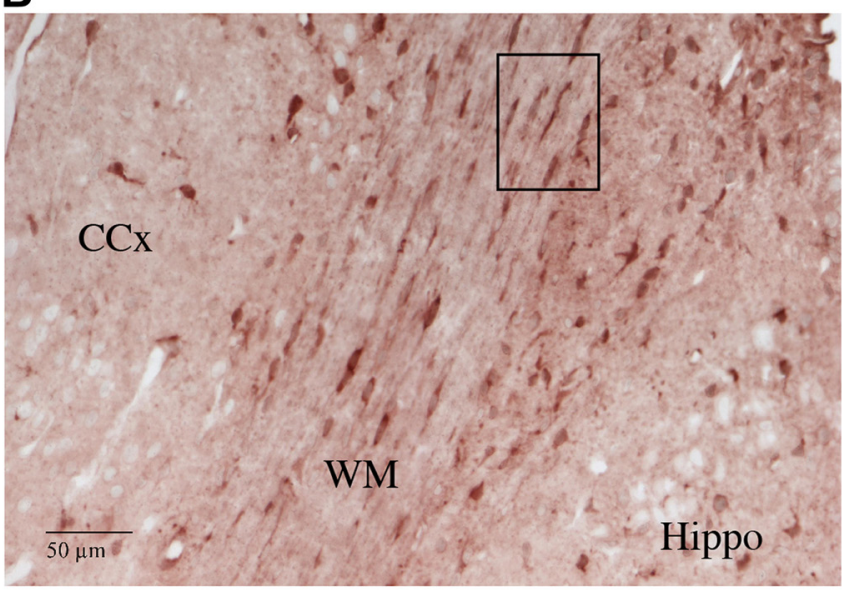

$\mathbf{E}$

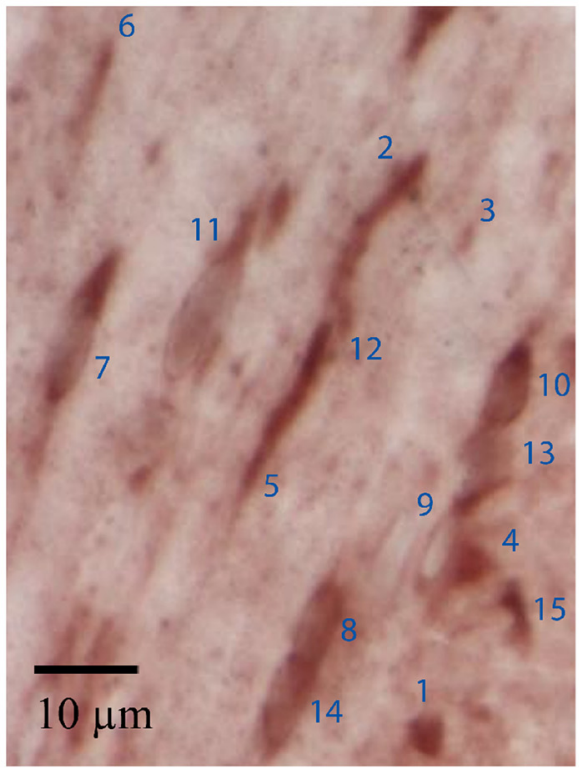

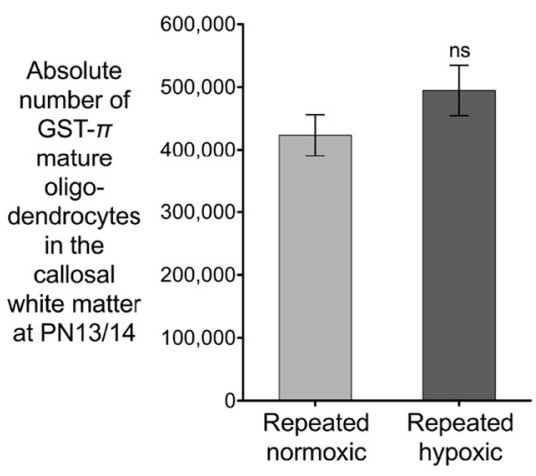

normoxic hypoxic

Figure 4. Rats exposed to repeated hypoxia from P1 to P3 did not have a significant loss of GST- $\pi$-positive mature oligodendrocytes in the callosal white matter at P13/P14.A-D, Images of the callosal white matter and adjacent brain regions from a repeated hypoxic brain. $A, C$, Reference section; $B, D$, Look-up section. CCx, Cerebral cortex; Hippo, hippocampus; WM, callosal white matter. The boxed areas in $\boldsymbol{A}$ and $\boldsymbol{B}$ are magnified in $\boldsymbol{C}$ and $\boldsymbol{D}$, respectively, to illustrate disector oligodendrocytes. Oligodendrocytes $1-6$ are disector cells for the reference section ( $\boldsymbol{C}$. 0 ligodendrocytes 7 -10 are disector cells for the look-up section (D). Because oligodendrocytes 11-14 have a nucleus in both sections and oligodendrocyte 15 has cytoplasm only in both sections, they were not counted as disector cells. $\boldsymbol{E}$, Absolute number of GST- $\pi$-positive mature oligodendrocytes. Two groups of rats were used: (1) repeated normoxic $(n=4)$ and (2) repeated hypoxic $(n=4)$ [paired two-tailed Student's $t$ test, not significant (ns), $p=0.364$ ]. Reliable estimates were obtained for the stereological data in this figure (see also the Results). Error bars show SEM.

estimates. The CE was calculated according to Gundersen et al. (1999) for the absolute volume and absolute number of neurons in the cerebral cortex and striatum, the absolute volume of the cerebral white matter and callosal white matter, and the absolute number of O4-positive pre-

$\leftarrow$

(Figure legend continued.) $\quad \boldsymbol{A}$ and $\boldsymbol{B}$. A representative axon from each sampled area in $\boldsymbol{A}$ and $\boldsymbol{B}$ is illustrated, respectively, at higher magnification in $\mathbf{C}$ and $\boldsymbol{D}$. C and $\boldsymbol{D}$ illustrate the measurements that were made to calculate the $g$-ratio. Specifically, the axon diameter was measured between the two innermost very short lines that have been drawn perpendicular to the short axis of the axon. The short axis of an axon was the maximum width perpendicular to the longest axis of an axon. The oligodendrocyte diameter and the myelin diameter were, respectively, measured between the two middle and the two outermost very short lines that have been drawn perpendicular to the short axis of the axon. $\boldsymbol{E}, g$-Ratio of myelinated axons. Two groups of rats were used: (1) repeated normoxic $(n=5)$ and (2) repeated hypoxic $(n=5)$ (paired two-tailed Student's $t$ test, $\left.{ }^{*} p=0.008\right)$. $\boldsymbol{F}$, Shrinkage-corrected number of myelinated axons per $100 \mu \mathrm{m}^{2}$. Same rats as $\boldsymbol{E}$ (paired two-tailed Student's $t$ test, ${ }^{*} p=$ 0.024). Reliable estimates were obtained for the stereological data in this figure (see also Results). Error bars show SEM. oligodendroglia and GST- $\pi$-positive mature oligodendrocytes in the callosal white matter. The $\mathrm{CE}$ of the numerical density (i.e., $N_{v}$ or number per subvolume) estimate for cortical neurons, striatal neurons, O4positive pre-oligodendroglia, and GST- $\pi$-positive mature oligodendrocytes was calculated as $1 /\left(\sum Q^{-}\right)^{1 / 2}$ (Larsen et al., 2004). The CE for the estimate of the reference volume and absolute surface area of myelin was calculated as described by Michel and Cruz-Orive (1988) using the revised formula of Gundersen et al. (1999). The CEs for the number of myelinated axons per $100 \mu \mathrm{m}^{2}$ and the diameter of myelinated axons were calculated as described previously (Partadiredja et al., 2003). For the mean somal volume of the striatal medium-spiny neurons, the CE was calculated as $\mathrm{CE}=\mathrm{SEM}(\ln (v))=\mathrm{CV} /\left(\sum \mathrm{Q}^{-}\right)^{1 / 2}$, where $v$ is the individual neuronal somal estimates for each rat, and $\mathrm{CV}$ is the coefficient of variation of these estimates within each rat (Dorph-Petersen et al., 2004).

Behavioral testing. Twenty rats (those used in the adult myelin study) were tested on a staircase test at 2.4 months of age and an eight-arm radial arm maze at 8.5 months of age (Fig. 5D). A modified version of the staircase test was used as described previously (Hobbs and Oorschot, 2008). For the radial arm maze, testing occurred from P259 to P266, with 
A

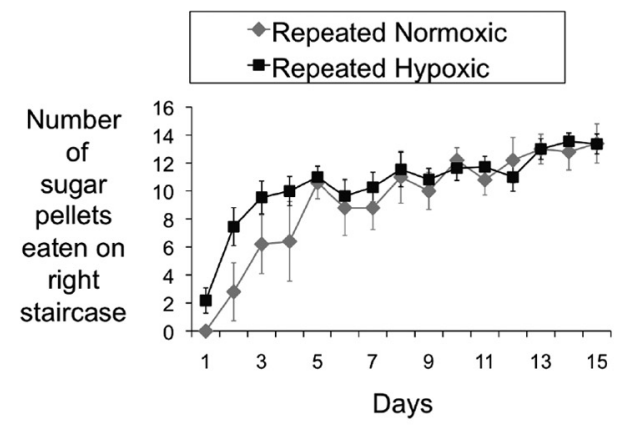

B

C

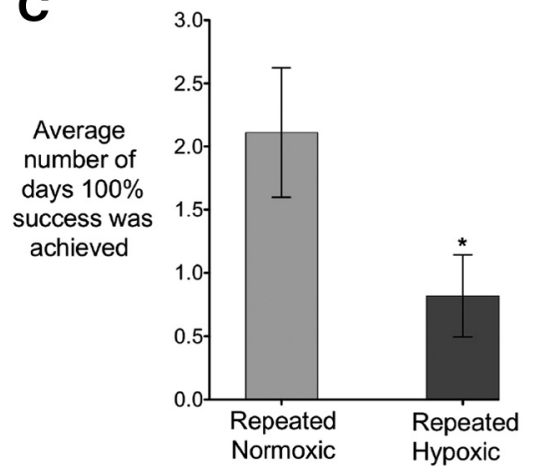

D

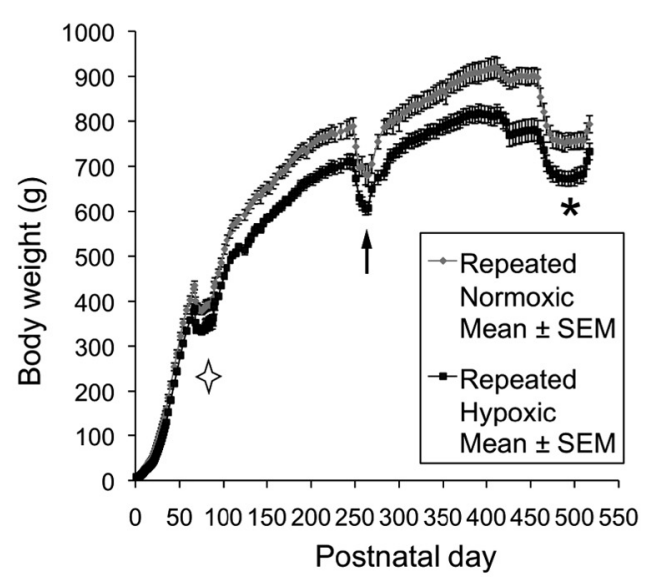

$\nLeftarrow$ food deprivation for staircase testing

$\uparrow$ food deprivation for radial arm maze testing

* food deprivation for testing ADHD-like hyperactivity

$\mathbf{E}$

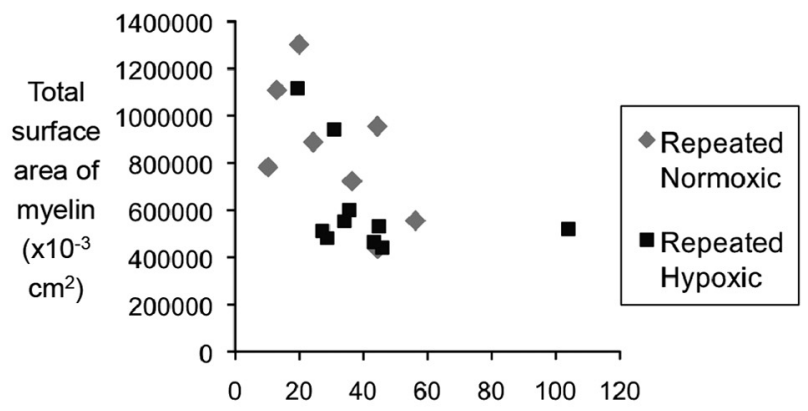

Score for ADHD-like hyperactivity

Figure 5. Rats exposed to repeated hypoxia from $P 1$ to $P 3$ had significant deficits in memory and body weight in adulthood but no motor deficits. For both repeated normoxic and repeated hypoxic rats, less cerebral myelin was associated with more ADHD-like hyperactivity. $A$, Number of sugar pellets retrieved with the right forelimb and then eaten on the staircase test at 2.4 months of age. Two groups of rats were used: (1) repeated normoxic $(n=5)$ and (2) repeated hypoxic $(n=11)$ (repeated-measures ANOVA, $p<0.601)$. The same result was observed for the left forelimb (data not shown). Four repeated normoxic rats were excluded from this analysis because of their enduring nonperformance (i.e., failure to eat and retrieve $>2$ sugar pellets per day from days $3-15$ of testing). $\boldsymbol{B}, \boldsymbol{C}$, Performance on the radial arm maze at 8.5 months of age. Two groups of rats were used: (1) repeated normoxic $(n=9)$ and (2) repeated hypoxic $(n=11)$. $\boldsymbol{B}$, Number of errors made per session (two-tailed Student's $t$ test, $\left.t_{(14)}=1.884, p=0.080\right)$. C, Number of days $100 \%$ success (i.e., no errors) was achieved (two-tailed Student's $t$ test, ${ }^{*} p=0.040$ ). The repeated normoxic rats made more correct choices before the first error ( $5.85 \pm 0.28)$ than the hypoxic animals $(5.13 \pm 0.27)$ and made less arm entries per session $(11.35 \pm 0.48)$ than the repeated hypoxic animals $(12.63 \pm 0.68)$, although both of these measures failed to reach statistical significance (two-tailed Student's test, $t_{(18)}=1.895, p=0.074$ and $t_{(18)}=1.523, p=0.145$, respectively). Neither the total time taken to complete the maze successfully nor the average time spent in each arm was statistically different between the two groups. $\boldsymbol{D}$, Body weight from P0 to P545 (0orschot et al., 2007 ). Same rats as in $\boldsymbol{A}-\boldsymbol{C}$ (for statistics, see Results). $\boldsymbol{E}$, Linear regression for the absolute (i.e., total) surface area of myelin at 18 months of age versus the degree of ADHD-like hyperactivity at 16 months of age (0orschot et al., 2007). Same rats as in $\boldsymbol{A}-\boldsymbol{D}$ (Pearson's correlation, $p=0.030$ ). The score for ADHD-like hyperactivity on the $x$-axis is the average score for each rat at the 11th 10-s segment of testing on the fixed-interval component of the operant test (0orschot et al., 2007, their Fig. 3). For A-D, error bars show SEM.

body weight maintained at $85 \%$ during testing (Liu and Bilkey, 1999, 2001). The apparatus consisted of a radial eight-arm maze constructed of clear Plexiglas and raised $\sim 60 \mathrm{~cm}$ off the ground. The arms were $70 \mathrm{~cm}$ long $\times 9 \mathrm{~cm}$ wide and radiated out from the center platform, which was $34 \mathrm{~cm}$ wide. The maze was surrounded by extramaze cues. Before testing, each rat received two $20 \mathrm{~min}$ sessions of habituation to the apparatus. In the first session, sugar pellets, Coco Pops (Kellogg's), and food pellets were used as rewards, scattered throughout the maze and placed at the ends of the arms so as to be readily visible. Then each rat was placed in the maze for $20 \mathrm{~min}$ to explore (Liu and Bilkey, 1999, 2001). The same procedure occurred on the second day, except that the only rewards were three sugar pellets placed at the end of arms so they would be visible from the center platform. If the rat successfully ate all the sugar pellets, then one more was placed in each arm to prevent the animal from becoming bored during the second day of habituation.

Rats were then tested every day for 8 consecutive days from P259 to P266. One sugar pellet was used as a reward but was hidden from view at the end of each arm. Each rat had a free choice of arms until they correctly chose all eight arms, were immobile for a period of $2 \mathrm{~min}$, or $10 \mathrm{~min}$ had passed since the beginning of the test. All arm entries made were recorded, as well as the time the rat entered each arm and the total time taken to complete the maze successfully (Liu and Bilkey, 1999, 2001; Paul et al., 2009). Errors were recorded as entering an arm that had been visited previously. Before testing each day, the maze was rotated three 
Table 1. Repeated hypoxia decreased brain weight

\begin{tabular}{|c|c|c|c|}
\hline Brain region & $\begin{array}{l}\text { Repeated } \\
\text { normoxia }\end{array}$ & $\begin{array}{l}\text { Repeated } \\
\text { hypoxia }\end{array}$ & $\begin{array}{l}\text { Percentage difference for } \\
\text { repeated normoxia versus } \\
\text { repeated hypoxia }\end{array}$ \\
\hline \multicolumn{4}{|c|}{ Left cerebral hemisphere } \\
\hline Mean & 0.857 & 0.822 & \multirow[t]{4}{*}{$4 \%$ reduction, NS } \\
\hline SD & 0.048 & 0.048 & \\
\hline SEM & 0.017 & 0.016 & \\
\hline $\mathrm{CV}$ & 0.056 & 0.058 & \\
\hline \multicolumn{4}{|c|}{ Right cerebral hemisphere } \\
\hline Mean & 0.919 & 0.870 & \multirow[t]{4}{*}{$5 \%$ reduction ${ }^{*}$} \\
\hline SD & 0.045 & 0.039 & \\
\hline SEM & 0.016 & 0.013 & \\
\hline $\mathrm{CV}$ & 0.049 & 0.045 & \\
\hline \multicolumn{4}{|l|}{ Hindbrain } \\
\hline Mean & 0.642 & 0.606 & \multirow[t]{4}{*}{$6 \%$ reduction ${ }^{* *}$} \\
\hline SD & 0.034 & 0.030 & \\
\hline SEM & 0.012 & 0.010 & \\
\hline $\mathrm{CV}$ & 0.053 & 0.050 & \\
\hline \multicolumn{4}{|l|}{ Whole brain } \\
\hline Mean & 2.415 & 2.293 & \multirow[t]{4}{*}{$5 \%$ reduction ${ }^{* * *}$} \\
\hline SD & 0.062 & 0.093 & \\
\hline SEM & 0.022 & 0.031 & \\
\hline CV & 0.026 & 0.041 & \\
\hline
\end{tabular}

Mean \pm SD of the weight of each brain structure and the percentage difference between male repeated hypoxic $(n=10)$ and repeated normoxic $(n=9)$ Sprague Dawley rats at $P 545$. Statistical data are for a two-tailed Student's $t$ test: left cerebral hemisphere, not statistically significant (NS), $t_{(17)}=1.480, p=0.157$; right cerebral hemisphere, $t_{(17)}=2.430,{ }^{*} p=0.026$; hindbrain, $t_{(17)}=2.417,{ }^{* *} p=0.027$; whole brain, $t_{(17)}=3.136,{ }^{* *} p=$ $0.006 . \mathrm{CV}=\mathrm{SD} /$ mean

arms clockwise to prevent the rats using intramaze cues to solve the maze. Also the maze was wiped out between each animal to prevent odor cues from being used.

After behavioral testing from 16 months of age (Fig. $5 D, E$ ) to investigate attention deficit hyperactivity disorder (ADHD)-like hyperactivity and inattention (Oorschot et al., 2007), these rats were anesthetized and intracardially perfused at P545 (i.e., 18 months of age). Each left hemisphere was then analyzed for the absolute surface area of myelin in the cerebral white matter, as described above.

Statistical analyses. Averages were expressed as mean \pm SEM and compared using a Student's $t$ test. Motor skills on the staircase test were compared using repeated-measures ANOVA. A possible association between the long-term myelin and behavioral data was analyzed using Pearson's correlation. $p<0.05$ was considered statistically significant.

\section{Results}

\section{Repeated hypoxia decreased body and brain weight}

Mortality from repeated hypoxic exposure was $<15 \%$ in all 13 litters. Repeated hypoxic rats had a lifelong deficit in body weight (Fig. 5D; Oorschot et al., 2007; P2: repeated hypoxia, $7.7 \pm 0.1 \mathrm{~g}$; repeated normoxia, $9.3 \pm 0.3 \mathrm{~g}$; two-tailed Student's $t$ test, $t_{(18)}=$ $5.518, p<0.0001 ; \mathrm{P} 509-\mathrm{P} 518$, which was the last day of testing for ADHD-like hyperactivity for all rats: repeated hypoxia, $674 \pm$ $12 \mathrm{~g}$; repeated normoxia, $761 \pm 19 \mathrm{~g}$; two-tailed Student's $t$ test, $\left.t_{(16)}=3.930, p=0.001\right)$. At P545, there was a statistically significant decrease in the weight of the whole brain, right cerebrum, and hindbrain in repeated hypoxic rats $(p<0.05$, two-tailed Student's $t$ test for all three brain regions; for the specific statistics for each brain region, see Table 1).

Repeated hypoxia decreased oligodendroglial precursor cell number, cerebral myelin, myelin per axon, myelinated axon number, and white matter volume but not the number of mature oligodendrocytes

At $\mathrm{P} 4$, the mean absolute number of O4-positive pre-oligodendrocytes in the callosal white matter was significantly reduced by $45 \%$ after repeated hypoxia (repeated hypoxia, $32,400 \pm 4000$; repeated normoxia, 58,700 \pm 6800; paired two-tailed Student's $t$ test, $t_{(3)}=3.775, p=0.032$; Fig. $2 B$ ). At P14, repeated hypoxia led to a statistically significant $29 \%$ decrease in the average absolute surface area of cerebral myelin (repeated hypoxia, 113,070 $\times$ $10^{-3} \pm 9094 \mathrm{~cm}^{2}$; repeated normoxia, $160,300 \times 10^{-3} \pm 17,127$ $\mathrm{cm}^{2}$; two-tailed Student's $t$ test, $t_{(14)}=2.604, p=0.021$; Fig. $2 F$ ). In the periventricular callosal white matter at P14/P15, repeated hypoxia led to less myelin per axon (i.e., a statistically significant $2 \%$ increase in the $g$-ratio of myelinated axons; repeated hypoxia, $0.8207 \pm 0.0088$; repeated normoxia, $0.8008 \pm 0.0061$; paired twotailed Student's $t$ test, $t_{(4)}=4.838, p=0.008$; Fig. $3 E$ ). Repeated hypoxia also led to a statistically significant $16 \%$ decrease in the shrinkage-corrected number of myelinated axons per $100 \mu \mathrm{m}^{2}$ of periventricular white matter (repeated hypoxia, $28.21 \pm 2.02$; repeated normoxia, $33.68 \pm 1.92$; paired two-tailed Student's $t$ test, $t_{(4)}=3.561, p=0.024 ;$ Fig. $\left.3 F\right)$. There was no significant difference in the shrinkage-corrected average diameter of myelinated axons in the periventricular callosal white matter at P14/P15 (repeated hypoxia, $0.671 \pm 0.041 \mu \mathrm{m}$; repeated normoxia, $0.641 \pm 0.027 \mu \mathrm{m}$; paired two-tailed Student's $t$ test, $t_{(4)}=0.535, p=0.621$ ). At P14, there was a statistically significant decrease in the total overall volume of cerebral white matter after repeated hypoxia (twotailed Student's $t$ test, $t_{(15)}=4.116, p<0.001$; Table 2). In the callosal white matter at P13/P14, there was no statistical difference in the absolute number of mature GST- $\pi$-positive mature oligodendrocytes for the repeated hypoxic versus repeated normoxic animals (repeated hypoxia, 494,791 \pm 46,144; repeated normoxia, $423,303 \pm 38,105$; paired twotailed Student's $t$ test, $t_{(3)}=1.067, p=0.364$; Fig. $4 E$ ).

Adult repeated hypoxic rats at 18 months of age had a statistically significant $25 \%$ loss in the absolute surface area of cerebral myelin (repeated hypoxia, $617,963 \times 10^{-3} \pm 75,203 \mathrm{~cm}^{2}$; repeated normoxia, $823,777 \times 10^{-3} \pm 96,263 \mathrm{~cm}^{2}$; one-tailed Student's $t$ test, $t_{(17)}=1.802, p=0.045$; Fig. $\left.2 G\right)$. Hence, an initial deficit in myelin in repeated hypoxic rats persists into adulthood (Figs. $2 \mathrm{~F}-\mathrm{H}$ ). For both groups, there was a statistically significant increase of $80-82 \%$ in the average total surface area of myelin between P14 and 18 months of age (repeated hypoxic rats at P14 compared with 18 months of age, two-tailed Student's $t$ test, $t_{(16)}=6.263, p<0.0001$; repeated normoxic rats at P14 compared with 18 months of age, two-tailed Student's $t$ test, $t_{(15)}=$ 6.786, $p<0.0001$; Fig. $2 H$ ).

\section{Repeated hypoxia decreased cerebral cortical and striatal volume with no neuronal loss or decreased striatal neuronal nuclear volume}

Repeated hypoxia led to a statistically significant $10-11 \%$ reduction in the absolute volume of the cerebral cortical gray matter and the striatum at P14 ( $p<0.01$ and $p<0.05$, respectively, two-tailed Student's $t$ test; for specific statistics, see Table 2). There was no significant difference in the absolute number of cerebral cortical neurons or striatal medium-spiny neurons between the repeated hypoxic and the repeated normoxic groups at P14 (Table 2). These statistical differences for the total volume of the cerebral cortex and striatum were also evident after correction for processing-induced shrinkage (data not shown). There was no difference in the shrinkage-corrected average nuclear volume of striatal medium-spiny neurons between repeated hypoxic $\left(809 \pm 36 \mu \mathrm{m}^{3}\right)$ and repeated normoxic $\left(833 \pm 28 \mu \mathrm{m}^{3}\right)$ rats at P14 (two-tailed Student's $t$ test, $t_{(14)}=0.548, p=0.592$ ). 
Table 2. Repeated hypoxia decreased cerebral white matter volume and cerebral grey matter volumes but not absolute neuronal number

\begin{tabular}{|c|c|c|c|c|}
\hline $\begin{array}{l}\text { Measured parameter and } \\
\text { experimental condition }\end{array}$ & $\begin{array}{l}\text { Rats } \\
(n)\end{array}$ & $\begin{array}{l}\text { Absolute } \\
\text { volume }\left(\mathrm{mm}^{3}\right)\end{array}$ & $\begin{array}{l}N_{v}\left(10^{4} / \mathrm{mm}^{3}\right) \\
\text { of neurons }\end{array}$ & $\begin{array}{l}N\left(10^{6}\right) \\
\text { of neurons }\end{array}$ \\
\hline \multicolumn{5}{|l|}{ Cerebral white matter } \\
\hline Repeated normoxia & 8 & & & \\
\hline Mean & & 8.94 & $\mathrm{~N} / \mathrm{A}$ & $\mathrm{N} / \mathrm{A}$ \\
\hline SD & & 0.45 & & \\
\hline SEM & & 0.17 & & \\
\hline CV & & 0.050 & & \\
\hline Repeated hypoxia & 9 & & & \\
\hline Mean & & $7.70^{* *}$ & $\mathrm{~N} / \mathrm{A}$ & $\mathrm{N} / \mathrm{A}$ \\
\hline SD & & 0.99 & & \\
\hline SEM & & 0.35 & & \\
\hline CV & & 0.129 & & \\
\hline \multicolumn{5}{|l|}{ Cerebral cortex } \\
\hline Repeated normoxia & 8 & & & \\
\hline Mean & & 92.48 & 15.01 & 13.88 \\
\hline SD & & 4.90 & 1.25 & 1.03 \\
\hline SEM & & 1.85 & 0.47 & 0.39 \\
\hline CV & & 0.053 & 0.083 & 0.074 \\
\hline Repeated hypoxia & 9 & & & \\
\hline Mean & & $81.68^{*}$ & $16.51^{* *}$ & 13.47 \\
\hline SD & & 8.56 & 1.40 & 1.53 \\
\hline SEM & & 3.03 & 0.50 & 0.54 \\
\hline $\mathrm{CV}$ & & 0.105 & 0.085 & 0.114 \\
\hline \multicolumn{5}{|l|}{ Striatum } \\
\hline Repeated normoxia & 8 & & & \\
\hline Mean & & 11.60 & 22.10 & 2.50 \\
\hline SD & & 0.79 & 1.06 & 0.11 \\
\hline SEM & & 0.30 & 0.40 & 0.04 \\
\hline CV & & 0.068 & 0.048 & 0.042 \\
\hline Repeated hypoxia & 9 & & & \\
\hline Mean & & $10.30^{* * *}$ & $25.60^{* * * *}$ & 2.60 \\
\hline SD & & 1.41 & 3.11 & 0.20 \\
\hline SEM & & 0.50 & 1.10 & 0.07 \\
\hline CV & & 0.137 & 0.122 & 0.076 \\
\hline
\end{tabular}

Mean \pm SD of the total number of cerebral cortical and striatal medium-spiny neurons and the total volume of the cerebral cortex, striatum, and cerebral white matter at $\mathrm{P} 14$ for repeated normoxic and repeated hypoxic rats. All data are for structures in the right cerebral hemisphere. N/A, Not applicable. Statistical data are for a two-tailed Student's $t$ test: cerebral white mattervolume, $t_{(15)}=4.116,{ }^{* *} p<0.001$; cerebral cortex volume, $t_{(15)}=4.026,{ }^{*} p=0.001$; striatal volume, $t_{(15)}=2.159 ;{ }^{* * *} p=0.048$; absolute neuronal number in cerebral cortex, $t_{(15)}=0.614, p=$ 0.548 ; absolute neuronal number in striatum, $t_{(15)}=1.198, p=0.249$. Because of a decreased absolute volume, the neuronal $N_{v}$ in both the cerebral cortex and the striatum was significantly increased after repeated hypoxia $\left(N_{v}\right.$ in cerebral cortex, $t_{(15)}=24.658,{ }^{* *} p<0.001 ; N_{v}$ in striatum, $\left.t_{(15)}=2.846,{ }^{* * * *} p=0.012\right)$. The statistical differences between repeated hypoxic and repeated normoxic rats for the absolute volume data and the neuronal $N$ in the cerebral cortex and striatum were also evident after correction for processing-induced shrinkage (data not shown). $(V=S D /$ mean.

Reliable stereological data

The mean CEs for the $N_{v}, N$, Vref, $S_{v}$, somal volume, axonal diameter, and axonal number measurements ranged from 1 to $10 \%$ (Tables 3-5). A mean CE of $10 \%$ or less is generally considered to yield a reliable estimate because the variation (or $\mathrm{CV}=$ $\mathrm{SD} /$ mean) between animals for the parameter being assessed is usually at least 10-15\% (Braendgaard et al., 1990). The observed mean variance for Vref and $S_{v}$ (i.e., $\mathrm{CE}^{2}$; Tables 3-5) was less than half of the total variance for each parameter for the respective group (i.e., $\mathrm{CV}^{2}$; Table 5). This indicated that most of the variation was attributable to biological variation rather than variation in the precision of the estimates made with the stereological techniques used (West and Gundersen, 1990; Oorschot, 1996; Gundersen et al., 1999). Reliable estimates were thus obtained for these parameters (West and Gundersen, 1990; Oorschot, 1996; Gundersen et al., 1999). When the $\mathrm{CE}^{2} / \mathrm{CV}^{2}$ ratio was not $<0.5$ (i.e., $0.89-3.56$ for $N_{v}$ or $N$ for striatal or cortical neurons; 0.76 for $N_{v}$ for mature oligodendrocytes; $0.55-1.02$ for $\ln (v)$ or somal volume; Table 5), there was a low biological variance (i.e., $\mathrm{CV}$;
Table 3. Final mean CE values of useful parameters from the left callosal white matter, right cerebral cortex, and right striatum of repeated normoxic and repeated normoxic rats based on the formula $\mathrm{CE}=\left(1 / n \times \Sigma_{i} \mathrm{CE}_{i}^{2}\right)^{1 / 2}$

\begin{tabular}{|c|c|c|c|}
\hline Measured parameter and experimental condition ${ }^{a}$ & $\mathrm{CEVref}^{b}$ & $\mathrm{CEN}_{v}{ }^{\mathrm{C}}$ & $\mathrm{CE} N^{d}$ \\
\hline \multicolumn{4}{|l|}{$\begin{array}{l}\text { Absolute number of callosal 04-positive } \\
\text { pre-oligodendrocytes, P4 }\end{array}$} \\
\hline Repeated normoxia & $\mathrm{N} / \mathrm{A}$ & 0.074 & 0.075 \\
\hline Repeated hypoxia & $\mathrm{N} / \mathrm{A}$ & 0.099 & 0.099 \\
\hline \multicolumn{4}{|l|}{$\begin{array}{c}\text { Absolute number of callosal GST- } \pi \text {-positive } \\
\text { mature oligodendrocytes, P13/P14 }\end{array}$} \\
\hline Repeated normoxia & 0.017 & 0.060 & 0.062 \\
\hline Repeated hypoxia & 0.017 & 0.052 & 0.056 \\
\hline \multicolumn{4}{|l|}{ Absolute number of cerebral cortical neurons, P14 } \\
\hline Repeated normoxia & 0.017 & 0.079 & 0.080 \\
\hline Repeated hypoxia & 0.018 & 0.080 & 0.080 \\
\hline \multicolumn{4}{|l|}{$\begin{array}{l}\text { Absolute number of striatal medium-spiny } \\
\text { neurons, P14 }\end{array}$} \\
\hline Repeated normoxia & 0.025 & 0.080 & 0.080 \\
\hline Repeated hypoxia & 0.027 & 0.079 & 0.079 \\
\hline
\end{tabular}

${ }^{a}$ To measure the absolute number of pre-oligodendrocytes, mature oligodendrocytes, or neurons, $8-15$ sections were analyzed per brain structure per rat. This generally fulfilled the guideline of sampling 10 or more sections per brain structure per animal (Gundersen et al., 1999).

${ }^{b}$ The mean total number of points counted to estimate the Vref ranged from 149 to 307 for the six groups. This exceeded the guideline of counting 100 points per structure (Gundersen et al., 1999).

'The mean number of disector pre-oligodendrocytes or disector neurons sampled per disector volume ranged from 0.50 to 1.42 for the six groups. The mean number of disector neurons was always more than one for the four groups. This generally complied with the guideline of sampling one to two cells per disector volume (West and Gundersen 1990). The mean number of disector mature oligodendrocytes sampled per disector volume, using the physical disector method, ranged from 10.55 to 12.04 for the two groups. Because the most time-consuming part of the physical disector analysis is matching the landmarks and cells in adjacent sections (0orschot, 1994), more disector cells were analyzed per sampling frame for this analysis.

${ }^{d}$ The mean total number of disector pre-oligodendrocytes or disector neurons sampled per structure ranged from 106 to 191 for the six groups. This complied with the guideline of counting 100-200 cells per brain structure per animal (Gundersen et al., 1988). The mean total number of disector mature oligodendrocytes sampled per callosal white matter ranged from 298 to 350 for the two groups. This exceeded this guideline.

Table 4. Final mean CE values of useful parameters from the cerebral white matter of repeated normoxic and repeated normoxic rats based on the formula $\mathrm{CE}=$ $\left(1 / n \times \Sigma_{i} \mathrm{CE}_{i}^{2}\right)^{1 / 2}$

\begin{tabular}{|c|c|c|}
\hline Measured parameter and experimental condition ${ }^{a}$ & CEVref $^{b}$ & $\mathrm{CESv^{c }}$ \\
\hline \multicolumn{3}{|l|}{ Absolute surface area of myelin, P14 } \\
\hline Repeated normoxia & 0.027 & 0.013 \\
\hline Repeated hypoxia & 0.032 & 0.016 \\
\hline \multicolumn{3}{|l|}{ Absolute surface area of myelin, 18 months old } \\
\hline Repeated normoxia & 0.019 & 0.015 \\
\hline Repeated hypoxia & 0.021 & 0.018 \\
\hline \multicolumn{3}{|l|}{ Absolute volume of cerebral white matter, P14 } \\
\hline Repeated normoxia & 0.018 & $\mathrm{~N} / \mathrm{A}$ \\
\hline Repeated hypoxia & 0.020 & N/A \\
\hline
\end{tabular}

${ }^{a}$ To measure the absolute surface area of myelin and the absolute volume of cerebral white matter, $10-12$ section were analyzed per rat. This fulfilled the guideline of sampling 10 or more sections per animal (Gundersen et al., 1999).

${ }^{b}$ The mean total number of points counted to estimate the absolute reference volume (Vref) ranged from 127 to 367 for the six conditions. This exceeded the guideline of counting 100 points per structure (Gundersen et al., 1999).

'The mean total number of intercepts counted to estimate the absolute surface volume $\left(S_{v}\right)$ ranged from 314 to 593 for the four conditions. This exceeded the guideline of counting 100 intercepts per structure (Michel and Cruz-Orive, 1988; Gundersen et al., 1999).

Braendgaard et al., 1990; Oorschot, 1996; Slomianka and West, 2005) for the specific parameter (i.e., $4.2-8.5 \%$ for the $N_{v}$ and $N$ data; $8.9-11.7 \%$ for somal volume; Table 5). A higher $\mathrm{CE}^{2} / \mathrm{CV}^{2}$ ratio is acceptable when the biological variance of a parameter is low or expected statistical outcomes are not jeopardized (Slomianka and West, 2005), as was evident in this study (e.g., neuronal $N$ and $N_{v}$ data, mature oligodendroglial $N_{v}$ data; Tables 2, 5).

Repeated hypoxia led to memory, but not motor, deficits At 2.4 months of age, repeated hypoxic rats showed no motor impairments on the staircase test (repeated-measures ANOVA, 
Table 5. Summary of the precision of the stereological measurements

\begin{tabular}{|c|c|c|c|}
\hline $\begin{array}{l}\text { Measured parameter and experimental } \\
\text { condition }\end{array}$ & $(\text { Mean CE })^{2}$ & $(\mathrm{CV})^{2 a}$ & $(\text { Mean } \mathrm{CE})^{2}<1 / 2(\mathrm{CV})^{2} ?$ \\
\hline \multicolumn{4}{|l|}{$\begin{array}{l}\text { Absolute number of 04-positive pre- } \\
\text { oligodendrocytes, P4 }\end{array}$} \\
\hline Repeated normoxia, $N_{v}{ }^{b}$ & $(0.074)^{2}$ & $(0.211)^{2}$ & Yes \\
\hline Repeated hypoxia, $N_{v}$ & $(0.099)^{2}$ & $(0.211)^{2}$ & Yes \\
\hline Repeated normoxia, $N$ & $(0.075)^{2}$ & $(0.214)^{2}$ & Yes \\
\hline Repeated hypoxia, $N$ & $(0.099)^{2}$ & $(0.199)^{2}$ & Yes \\
\hline \multicolumn{4}{|l|}{ Absolute surface area of myelin, P14 } \\
\hline Repeated normoxia, Vref & $(0.027)^{2}$ & $(0.159)^{2}$ & Yes \\
\hline Repeated hypoxia, Vref & $(0.032)^{2}$ & $(0.194)^{2}$ & Yes \\
\hline Repeated normoxia, $S_{v}$ & $(0.013)^{2}$ & $(0.155)_{2}^{2}$ & Yes \\
\hline Repeated hypoxia, $S_{v}$ & $(0.016)^{2}$ & $(0.157)^{2}$ & Yes \\
\hline \multicolumn{4}{|l|}{$\begin{array}{l}\text { Absolute surface area of myelin, } 18 \\
\text { months old }\end{array}$} \\
\hline Repeated normoxia, Vref & $(0.019)^{2}$ & $(0.184)^{2}$ & Yes \\
\hline Repeated hypoxia, Vref & $(0.021)^{2}$ & $(0.288)^{2}$ & Yes \\
\hline Repeated normoxia, $S_{v}$ & $(0.015)^{2}$ & $(0.132)^{2}$ & Yes \\
\hline Repeated hypoxia, $S_{v}$ & $(0.018)^{2}$ & $(0.110)^{2}$ & Yes \\
\hline \multicolumn{4}{|c|}{ Number of myelinated axons, P14/P15 } \\
\hline Repeated normoxia & $(0.044)^{2}$ & $(0.114)^{2}$ & Yes \\
\hline Repeated hypoxia & $(0.051)^{2}$ & $(0.143)^{2}$ & Yes \\
\hline \multicolumn{4}{|l|}{$\begin{array}{l}\text { Diameter of myelinated axons, P14/ } \\
\text { P15' }\end{array}$} \\
\hline Repeated normoxia & $(0.062)^{2}$ & $(0.104)^{2}$ & Yes \\
\hline Repeated hypoxia & $(0.074)^{2}$ & $(0.122)^{2}$ & Yes \\
\hline \multicolumn{4}{|l|}{$\begin{array}{l}\text { Absolute volume of cerebral white } \\
\text { matter, P14 }\end{array}$} \\
\hline Repeated normoxia, Vref & $(0.018)^{2}$ & $(0.050)^{2}$ & Yes \\
\hline Repeated hypoxia, Vref & $(0.020)^{2}$ & $(0.129)^{2}$ & Yes \\
\hline \multicolumn{4}{|l|}{$\begin{array}{l}\text { Absolute number of GST- } \pi \text {-positive } \\
\text { mature oligodendrocytes, P13/ } \\
\text { P14 }\end{array}$} \\
\hline Repeated normoxia, Vref & $(0.017)^{2}$ & $(0.114)^{2}$ & Yes \\
\hline Repeated hypoxia, Vref & $(0.017)^{2}$ & $(0.106)^{2}$ & Yes \\
\hline Repeated normoxia, $N_{v}^{b}$ & $(0.060)^{2}$ & $(0.069)^{2}$ & No, $6.9 \% \mathrm{CV}$ is low ${ }^{d}$ \\
\hline Repeated hypoxia, $N_{v}$ & $(0.052)^{2}$ & $(0.250)^{2}$ & Yes \\
\hline Repeated normoxia, $N$ & $(0.062)^{2}$ & $(0.156)^{2}$ & Yes \\
\hline Repeated hypoxia, $N$ & $(0.056)^{2}$ & $(0.162)^{2}$ & Yes \\
\hline \multicolumn{4}{|l|}{$\begin{array}{l}\text { Absolute number of cerebral cortical } \\
\text { neurons, P14 }\end{array}$} \\
\hline Repeated normoxia, Vref & $(0.017)^{2}$ & $(0.053)^{2}$ & Yes \\
\hline Repeated hypoxia, Vref & $(0.018)^{2}$ & $(0.105)^{2}$ & Yes \\
\hline Repeated normoxia, $N_{v}$ & $(0.079)^{2}$ & $(0.083)^{2}$ & No, $8.3 \% \mathrm{CV}$ is low ${ }^{d}$ \\
\hline Repeated hypoxia, $N_{v}$ & $(0.080)^{2}$ & $(0.085)^{2}$ & No, $8.5 \% \mathrm{CV}$ is $\mathrm{low}^{d}$ \\
\hline Repeated normoxia, $N$ & $(0.080)^{2}$ & $(0.074)^{2}$ & No, $7.4 \% \mathrm{CV}$ is low ${ }^{d}$ \\
\hline Repeated hypoxia, $N$ & $(0.080)^{2}$ & $(0.114)^{2}$ & Yes \\
\hline \multicolumn{4}{|l|}{$\begin{array}{l}\text { Absolute number of striatal medium- } \\
\text { spiny neurons, P14 }\end{array}$} \\
\hline Repeated normoxia, Vref & $(0.025)^{2}$ & $(0.068)^{2}$ & Yes \\
\hline Repeated hypoxia, Vref & $(0.027)^{2}$ & $(0.137)^{2}$ & Yes \\
\hline Repeated normoxia, $N_{v}$ & $(0.080)^{2}$ & $(0.048)^{2}$ & No, $4.8 \% \mathrm{CV}$ is low ${ }^{d}$ \\
\hline Repeated hypoxia, $N_{v}$ & $(0.079)^{2}$ & $(0.122)^{2}$ & Yes \\
\hline Repeated normoxia, $N$ & $(0.080)^{2}$ & $(0.042)^{2}$ & No, $4.2 \% \mathrm{CV}$ is low ${ }^{d}$ \\
\hline Repeated hypoxia, $N$ & $(0.079)^{2}$ & $(0.076)^{2}$ & No, $7.6 \% \mathrm{CV}$ is low ${ }^{d}$ \\
\hline \multicolumn{4}{|l|}{$\begin{array}{l}\text { Somal volume of striatal medium-spiny } \\
\text { neurons, P14 }\end{array}$} \\
\hline Repeated normoxia, $\ln (v)$ & $(0.082)^{2}$ & $(0.089)^{2}$ & No, $8.9 \% \mathrm{CV}_{\text {is }}$ low $^{d}$ \\
\hline Repeated hypoxia, $\ln (v)$ & $(0.087)^{2}$ & $(0.117)^{2}$ & $\begin{array}{l}\text { No, } 11.7 \% \text { CV is relatively } \\
\text { low }^{d}\end{array}$ \\
\hline
\end{tabular}

${ }^{a} \mathrm{CV}=\mathrm{SD} /$ mean.

${ }^{b}$ For an explanation of the abbreviations used, see Materials and Methods.

The total number of axons measured per animal ranged from 92 to 163 . The average was 129 measured axons per repeated normoxic animal and 113 measured axons per repeated hypoxic animal.

${ }^{d}$ For comments, see Results.
$F_{(1,14)}=0.287, p<0.601 ;$ Fig. $\left.5 A\right)$. Repeated hypoxic rats tested at 8.5 months of age achieved a score of $100 \%$ success (i.e., no errors) on the radial arm maze on significantly fewer days during the $8 \mathrm{~d}$ of testing (repeated hypoxia, $0.82 \pm 0.34 \mathrm{~d}$; repeated normoxia, $2.11 \pm 0.54 \mathrm{~d}$; two-tailed Student's $t$ test, $t_{(18)}=2.209$, $p=0.040$; Fig. $5 B, C)$. During the initial training of the animals on the radial arm maze, repeated hypoxic and repeated normoxic rats reached criterion at the same rates. Thus, repeated hypoxic rats did not learn more slowly. Specifically, the average number of days taken to achieve five or more correct responses in the first eight arm entries was $1.45 \pm 0.22 \mathrm{~d}$ (mean \pm SEM) for the repeated hypoxic rats and $1.44 \pm 0.23 \mathrm{~d}$ for the repeated normoxic rats (two-tailed Student's $t$ test, $t_{(18)}=0.032, p=0.975$ ). During the testing of the animals over $8 \mathrm{~d}$, the animals retained their learning of the task. Specifically, the average number of correct choices before the first error did not differ between repeated hypoxic and repeated normoxic rats on days 1 and 8 of testing (day 1: repeated hypoxia, $5.09 \pm 0.50$ correct choices before the first error vs $5.22 \pm 0.52$ for repeated normoxia, two-tailed Student's $t$ test, $t_{(18)}=0.190, p=0.851$; day 8: repeated hypoxia, $4.91 \pm$ 0.48 correct choices before the first error vs $5.56 \pm 0.50$ for repeated normoxia, two-tailed Student's $t$ test, $t_{(18)}=0.976, p=$ 0.342 ). There were also no significant differences when the days 1 and 8 results were compared for repeated hypoxic animals alone and for repeated normoxic animals alone (repeated hypoxic, two-tailed Student's $t$ test, $t_{(20)}=0.276, p=0.786$; repeated normoxic, two-tailed Student's $t$ test, $t_{(16)}=0.487, p=0.633$ ).

\section{Decreased cerebral myelin is correlated with increased ADHD-like hyperactivity}

A statistically significant negative correlation/linear regression was observed for the absolute surface area of cerebral myelin at 18 months of age versus the degree of ADHD-like hyperactivity at 16 months of age (Pearson's $r=0.51, p=0.030$; Fig. 5E; ADHD-like hyperactivity data from Oorschot et al., 2007). Hence, a lower myelin value was correlated with a higher score for ADHD-like hyperactivity.

\section{Discussion}

We demonstrate for the first time the induction of a rat brain and behavioral phenotype that mimics human extreme prematurity. The rat phenotype was induced in males by exposure to repeated hypoxia over $3 \mathrm{~d}$ during the second-trimester equivalent of extreme prematurity. This model mimics the short- and long-term white matter neuropathology seen in infants and older children that were born extremely prematurely. A loss of gray matter volume, without neuronal loss in the cerebral cortex and striatum, also mimics the brain injury seen in extreme prematurity. Memory deficits, without major motor deficits, were observed in the repeated hypoxic rats, which mimics behavioral outcomes of extreme prematurity. The animals used in this study for the longterm behavioral testing of memory and motor skills have also been tested for ADHD-like hyperactivity using a multiplecomponent fixed-interval extinction test (Oorschot et al., 2007). We found that adult male rats exposed to repeated hypoxia during the equivalent of extreme prematurity exhibit ADHD-like hyperactivity in response to delayed reward (Oorschot et al., 2007). The most common behavioral disorder seen in children and adolescents born extremely prematurely is ADHD (Halsey et al., 1996). Together, these findings indicate the remarkable similarity of the rat brain and behavioral phenotype to that observed after human extreme prematurity. 
A major advantage of this new rat model is that it mimics the clinical context of human extreme prematurity at multiple levels. These include the use of a developmental brain age that is akin to the human, the use of repeated hypoxia rather than chronic (Mikati et al., 2005) or acute (Back et al., 2002) hypoxia, and global brain hypoxia is mimicked in contrast to unilateral brain injury (Back et al., 2002). The novel rat model exposes immature rat pups to repeated hypoxia from $\mathrm{P} 1$ to $\mathrm{P} 3$. The $\mathrm{P} 1-\mathrm{P} 3$ rat brain is very similar to the human brain at 24-26 weeks of gestation in terms of amino acid neurotransmitter maturation, white matter maturation, oligodendroglial lineage, and cortical and axonal development (Hagberg et al., 1997; Balasubramaniam and Del Bigio, 2006). Other major advantages (Chapados and Cheung, 2008) of the new rat model include a survivability that permits long-term behavioral and pathological analyses, the reproducibility of the observed deficits, and an association between a behavioral and anatomical result (i.e., ADHD-like hyperactivity is correlated with decreased cerebral myelin). There is a paucity of long-term neuropathological and behavioral data in the currently available rodent models of hypoxic brain injury during the second-trimester equivalent (Scafidi et al., 2009).

In large-animal models, such as the fetal sheep and fetal baboon, comparable studies measuring long-term memory, motor skills, and ADHD-like hyperactivity do not appear to be published. Although large-animal studies are useful for obtaining physiological measures, the prohibitive costs and difficulties of behavioral testing and long-term assessment of neuropathology in these models means that they are largely unsuitable for investigating mechanisms of injury and new treatment options in the first instance (Scafidi et al., 2009). Relevant long-term behavioral and pathological effects occur in the rat model, even though the gray matter/white matter ratio in the brain of larger animals (e.g., sheep) is more similar to humans (Silbereis et al., 2010).

A loss of O4-positive pre-oligodendrocytes and/or a halt in their progression into mature myelin-producing cells is likely to be responsible for the ongoing white matter volume loss and impaired myelination detected in infants, children, and adolescents born extremely prematurely (Volpe, 2009). It is highly likely that the loss of white matter volume and the long-term decrease in the surface area of myelin in this rat model result from the decreased number of O4-positive pre-oligodendrocytes at $\mathrm{P} 4$.

The absolute number of mature oligodendrocytes was restored by $\mathrm{P} 13 / \mathrm{P} 14$, yet myelin deficits were evident. These findings mimic abnormalities in myelin expression, without an overt loss of mature oligodendrocytes, seen in extreme prematurity (Billiards et al., 2008). Mature oligodendrocytes at P13/P14 may be regenerated from stem cells in the subventricular zone (Levison et al., 2001), yet they were unable to restore myelination. After P14, rats exposed to repeated hypoxia during P1-P3 have a normal capacity for producing myelin. They had a similar increase in myelin from P14 to 18 months of age as the normoxic group. This suggests that damage to the myelin system occurs in the first 2 weeks postnatally, directly after exposure to repeated hypoxia. Detailed characterization of oligodendrocytic development and myelination during P0-P14 will have important implications for preventing myelin deficits after repeated hypoxia.

Myelin and O4-positive pre-oligodendrocytes were measured in the left and right cerebral hemispheres, respectively. Myelin was examined in the left cerebrum because dopaminergic deficits occur specifically in the right cerebrum (Brake et al., 2000; Arnsten, 2009). Dopaminergic analyses of the right cerebrum are the focus of a parallel study. Based on the literature, an asymmetry in the hemispheric response for oligodendrocytes and myelin is unlikely (Sahin et al., 2001; Partadiredja et al., 2003).

The white matter damage and hypomyelination that occurs in diffuse PVL in human infants is thought to contribute to the high incidence of memory and learning deficits (Ment et al., 2009), as well as other neurodevelopmental disorders, seen in children and adolescents who were born extremely prematurely. At 16 years of age, deficits in visuospatial reasoning and working memory are still evident (Taylor et al., 2004). The radial arm maze is a test for spatial learning ability that measures working memory (Paul et al., 2009). Performance on the radial arm maze was impaired in adult repeated hypoxic rats. It is likely that decreased myelination and white matter volume in these rats is contributing to the cognitive impairment detected on this task. This could result from slower conductance velocities in myelinated axons attributable to the observed thinner myelin and decreased number of myelinated axons. Possible associations between white matter and memory deficits were not undertaken in this study because these specific measures were undertaken 10 months apart to accommodate measurement of ADHD-like hyperactivity (Oorschot et al., 2007).

A reduction in cerebral white matter volume is common in humans with ADHD (Castellanos et al., 2002). Thus, the white matter volume and myelination deficits seen in repeated hypoxic rats may also be related to the development of ADHD-like hyperactivity, as well as deficient cognitive processing. We report here that a decreased absolute surface area of cerebral myelin was significantly correlated with a higher score for ADHD-like hyperactivity (Oorschot et al., 2007). This is the first evidence in animals that myelin deficits are associated with ADHD-like hyperactivity.

Potential hypotheses for the volume loss, in the absence of neuronal loss, in the cerebral cortex and the striatum in repeated hypoxic rats may include decreased arborization of axons and/or glial cells in these structures. For the striatum, data from this study indicate that it is unlikely to be attributable to a deficit in the average nuclear volume of the spiny projection neurons, which constitute $>97 \%$ of rat striatal neurons (Oorschot et al., 2002).

Male children born extremely prematurely have a long-term deficit in body weight that is evident in adolescence and at 20 years of age (Hack et al., 2003). Modern neuroimaging techniques in infants and children consistently report a decreased volume of the cerebrum and cerebellum for infants with diffuse white matter injury (Ment et al., 2009). A long-term deficit in body weight and in the weight of the cerebrum and hindbrain, including the cerebellum, were observed in male repeated hypoxic rats at 18 months of age. These findings provide additional evidence that the rat model mimics human extreme prematurity.

Future work using this model should investigate the following: (1) the biological basis for a long-term deficit in body weight; (2) potential associations between myelin loss, global white matter loss, and either memory loss (Northam et al., 2011) or diffusion tensor imaging (Tao et al., 2012); (3) whether reactive/ hypertrophied astrocytes are evident in the cerebral white, but not gray, matter at later stages of diffuse brain injury because this is evident in humans born extremely prematurely (Golden et al., 1997; Haynes et al., 2003; Back et al., 2007); and (4) the effect of infection on repeated hypoxic rats because the animals were healthy in this animal model, whereas most infants born extremely prematurely are born very sick with attendant systemically elevated levels of cytokines (Matoba et al., 2009).

In summary, a novel rat model has been discovered that closely mimics the short- and long-term brain injury found in 
infants born extremely prematurely. This includes a loss of oligodendroglial precursor cells, decreased white matter volume, hypomyelination, and gray matter volume loss without overt neuronal loss in the cerebral cortex and striatum. It also includes spatial memory impairment on the radial arm maze, without motor deficits on the staircase test, and long-term deficits in myelination that persist into adulthood. Previously, we reported ADHD-like hyperactivity in adult rats after repeated hypoxic exposure during development (Oorschot et al., 2007). Here we report that myelin deficits are correlated with ADHD-like hyperactivity. This rat model will provide a valuable new tool to investigate treatment interventions aimed at preventing or ameliorating the spectrum of brain injuries and associated deficits seen in children born extremely prematurely.

\section{References}

Arnsten AF (2009) Toward a new understanding of attention-deficit hyperactivity disorder pathophysiology: an important role for prefrontal cortex dysfunction. CNS Drugs 23 [Suppl 1]:33-41. CrossRef Medline

Back SA, Han BH, Luo NL, Chricton CA, Xanthoudakis S, Tam J, Arvin KL, Holtzman DM (2002) Selective vulnerability of late oligodendrocyte progenitors to hypoxia-ischemia. J Neurosci 22:455-463. Medline

Back SA, Riddle A, McClure MM (2007) Birth maturation-dependent vulnerability of perinatal white matter in premature birth. Stroke 38:724730. CrossRef Medline

Baddeley AJ, Gundersen HJ, Cruz-Orive LM (1986) Estimation of surface area from vertical sections. J Microsc 142:259-276. CrossRef Medline

Balasubramaniam J, Del Bigio MR (2006) Animal models of germinal matrix hemorrhage. J Child Neurol 21:365-371. CrossRef Medline

Billiards SS, Haynes RL, Folkerth RD, Borenstein NS, Trachtenberg FL, Rowitch DH, Ligon KL, Volpe JJ, Kinney HC (2008) Myelin abnormalities without oligodendrocyte loss in periventricular leukomalacia. Brain Pathol 18:153-163. CrossRef Medline

Braendgaard H, Evans SM, Howard CV, Gundersen HJ (1990) The total number of neurons in the human neocortex unbiasedly estimated using optical disectors. J Microsc 157:285-304. CrossRef Medline

Brake WG, Sullivan RM, Gratton A (2000) Perinatal distress leads to lateralized medial prefrontal cortical dopamine hypofunction in adult rats. J Neurosci 20:5538-5543. Medline

Castellanos FX, Lee PP, Sharp W, Jeffries NO, Greenstein DK, Clasen LS, Blumenthal JD, James RS, Ebens CL, Walter JM, Zijdenbos A, Evans AC, Giedd JN, Rapoport JL (2002) Developmental trajectories of brain volume abnormalities in children and adolescents with attention-deficit/ hyperactivity disorder. JAMA 288:1740-1748. CrossRef Medline

Chapados I, Cheung PY (2008) Not all models are created equal: animal models to study hypoxic-ischemic encephalopathy of the newborn. Neonatology 94:300-303. CrossRef Medline

Dorph-Petersen KA, Pierri JN, Sun Z, Sampson AR, Lewis DA (2004) Stereological analysis of the mediodorsal thalamic nucleus in schizophrenia: volume, neuron number, and cell types. J Comp Neurol 472:449-462. CrossRef Medline

Golden JA, Gilles FH, Rudelli R, Leviton A (1997) Frequency of neuropathological abnormalities in very low birth weight infants. J Neuropath Exp Neurol 56:472-478. CrossRef Medline

Gundersen HJ (1977) Notes on the estimation of numerical density of arbitrary profiles: the edge effect. J Microsc 111:219-223. CrossRef

Gundersen HJ, Bagger P, Bendtsen TF, Evans SM, Korbo L, Marcussen N, Møller A, Nielsen K, Nyengaard JR, Pakkenberg B, Sorenson FB, Vesterby A, West MJ (1988) The new stereological tools: disector, fractionator, nucleator and point sampled intercepts and their use in pathological research and diagnosis. APMIS 96:857-881. CrossRef Medline

Gundersen HJ, Jensen EB, Kiêu K, Nielsen J (1999) The efficiency of systematic sampling in stereology-reconsidered. J Microsc 193:199-211. CrossRef Medline

Hack M, Schluchter M, Cartar L, Rahman M, Cuttler L, Borawski E (2003) Growth of very low birth weight infants to age 20 years. Pediatrics 112: e30-e38. CrossRef Medline

Hagberg H, Bona E, Gilland E, Puka-Sundvall M (1997) Hypoxia-ischaemia model in the 7-day-old rat: possibilities and shortcomings. Acta Paediatr [Suppl] 422:85-88.

Halsey CL, Collin MF, Anderson CL (1996) Extremely low-birth-weight children and their peers. A comparison of school-age outcomes. Arch Pediatr Adolesc Med 150:790-794. CrossRef Medline

Haynes RL, Folkerth RD, Keefe RJ, Sung I, Swzeda LI, Rosenberg PA, Volpe JJ, Kinney HC (2003) Nitrosative and oxidative injury to premyelinating oligodendrocytes in periventricular leukomalacia. J Neuropathol Exp Neurol 62:441-450. Medline

Hobbs CE, Oorschot DE (2008) Neonatal rat hypoxia-ischemia: long-term rescue of striatal neurons and motor skills by combined antioxidanthypothermia treatment. Brain Pathol 18:443-454. CrossRef Medline

Johnson S (2007) Cognitive and behavioural outcomes following very preterm birth. Semin Fetal Neonatal Med 12:363-373. CrossRef Medline

Larsen M, Bjarkam CR, Østergaard K, West MJ, Sørensen JC (2004) The anatomy of the porcine subthalamic nucleus evaluated with immunohistochemistry and design-based stereology. Anat Embryol (Berl) 208:239-247. CrossRef Medline

Levison SW, Rothstein RP, Romanko MJ, Snyder MJ, Meyers RL, Vannucci SJ (2001) Hypoxia/ischemia depletes the rat perinatal subventricular zone of oligodendrocyte progenitors and neural stem cells. Dev Neurosci 23: 234-247. CrossRef Medline

Liu P, Bilkey DK (1999) The effect of excitotoxic lesions centered on the perirhinal cortex in two versions of the radial arm maze task. Behav Neurosci 113:672-682. CrossRef Medline

Liu P, Bilkey DK (2001) The effect of excitotoxic lesions centered on the hippocampus or perirhinal cortex in object recognition and spatial memory tasks. Behav Neurosci 115:94-111. CrossRef Medline

Lou HC (1996) Etiology and pathogenesis of ADHD: significance of prematurity and perinatal hypoxic-haemodynamic encephalopathy. Acta Paediatrica 85:1266-1271. CrossRef Medline

Matoba N, Yu Y, Mestan K, Pearson C, Ortiz K, Porta N, Thorsen P, Skogstrand K, Hougaard DM, Zuckerman B, Wang X (2009) Differential patterns of 27 cord blood immune biomarkers across gestational age. Pediatrics 123:1320-1328. CrossRef Medline

Ment LR, Hirtz D, Hüppi PS (2009) Imaging biomarkers of outcome in the developing preterm brain. Lancet Neurol 8:1042-1055. CrossRef Medline

Michel RP, Cruz-Orive LM (1988) Application of the Cavalieri principle and vertical sections method to the lung: estimation of volume and pleural surface area. J Microsc 150:117-136. CrossRef Medline

Mikati MA, Zeinieh MP, Kurdi RM, Harb SA, El Hokayem JA, Daderian RH, Shamseddine A, Obeid M, Bitar FF, El Sabban M (2005) Long-term effects of acute and of chronic hypoxia on behavior and on hippocampal histology in the developing brain. Brain Res Dev Brain Res 157:98-102. CrossRef Medline

Northam GB, Liégeois F, Chong WK, Wyatt JS, Baldeweg T (2011) Total brain white matter is a major determinant of IQ in adolescents born preterm. Ann Neurol 69:702-711. CrossRef Medline

Oorschot DE (1994) Are you using neuronal densities, synaptic densities or neurochemical densities as your definitive data? There is a better way to go. Prog Neurobiol 44:233-247. CrossRef Medline

Oorschot DE (1996) The total number of neurons in the neostriatal, pallidal, subthalamic and substantia nigral nuclei of the rat basal ganglia: a stereological study using the Cavalieri and optical disector methods. J Comp Neurol 366:580-599. CrossRef Medline

Oorschot DE, Tunstall MJ, Wickens JR (2002) Local connectivity between striatal spiny projection neurons: a re-evaluation. In: The basal ganglia VII (Nicholson L, Faull RLM, eds), pp 421-434. New York: Plenum.

Oorschot DE, Voss L, Covey MV, Bilkey DK, Saunders SE (2007) ADHDlike hyperactivity, with no attention deficit, in adult rats after repeated hypoxia during the equivalent of extreme prematurity. J Neurosci Methods 166:315-322. CrossRef Medline

Partadiredja G, Miller R, Oorschot DE (2003) The number, size, and type of axons in rat subcortical white matter on left and right sides: a stereological, ultrastructural study. J Neurocytol 32:1165-1179. CrossRef Medline

Paul CM, Magda G, Abel S (2009) Spatial memory: theoretical basis and comparative review on experimental methods in rodents. Behav Brain Res 203:151-164. CrossRef Medline

Paxinos G, Watson C (1998) The rat brain in stereotaxic coordinates, Ed 4. San Diego: Academic.

Paxinos G, Watson C (2007) The rat brain in stereotaxic coordinates, Ed 6. Amsterdam: Academic.

Poets CF, Stebbens VA, Richard D, Southall DP (1995) Prolonged episodes of hypoxemia in preterm infants undetectable by cardiorespiratory monitors. Pediatrics 95:860-863. Medline 
Sahin B, Aslan H, Unal B, Canan S, Bilgic S, Kaplan S, Tumkaya L (2001) Brain volumes of the lamb, rat and bird do not show hemispheric asymmetry: a stereological study. Image Anal Stereol 20:9-13. CrossRef

Scafidi J, Fagel DM, Ment LR, Vaccarino FM (2009) Modeling premature brain injury and recovery. Int J Dev Neurosci 27:863-871. CrossRef Medline

Silbereis JC, Huang EJ, Back SA, Rowitch DH (2010) Towards improved animal models of neonatal white matter injury associated with cerebral palsy. Dis Model Mech 3:678-688. CrossRef Medline

Slomianka L, West MJ (2005) Estimators of the precision of stereological estimates: an example based on the CA1 pyramidal cell layer of rats. Neuroscience 136:757-767. CrossRef Medline

Tandrup T (1993) A method for unbiased and efficient estimation of number and mean volume of specified neuronal subtypes in rat dorsal root ganglion. J Comp Neurol 329:269-276. CrossRef Medline

Tao JD, Barnette AR, Griffith JL, Neil JJ, Inder TE (2012) Histopathologic correlation with diffusion tensor imaging after chronic hypoxia in the immature ferret. Pediatr Res 71:192-198. CrossRef Medline

Taylor HG, Minich N, Bangert B, Filipek PA, Hack M (2004) Long-term neuropsychological outcomes of very low birth weight: associations with early risks for periventricular brain insults. J Int Neuropsychol Soc 10: 987-1004. Medline

Tos P, Ronchi G, Nicolino S, Audisio C, Raimondo S, Fornaro M, Battiston B, Graziani A, Perroteau I, Geuna S (2008) Employment of the mouse median nerve model for the experimental assessment of peripheral nerve regeneration. J Neurosci Methods 169:119-127. CrossRef Medline

Vederhus BJ, Markestad T, Eide GE, Graue M, Halvorsen T (2010) Health related quality of life after extremely preterm birth: a matched controlled cohort study. Health Qual Life Outcomes 8:53. CrossRef Medline

Volpe JJ (2009) Brain injury in premature infants: a complex amalgam of destructive and developmental disturbances. Lancet Neurol 8:110-124. CrossRef Medline

Volpe JJ, Kinney HC, Jensen FE, Rosenberg PA (2011) The developing oligodendrocyte: key cellular target in brain injury in the premature infant. Int J Dev Neurosci 29:423-440. CrossRef Medline

West MJ, Gundersen HJ (1990) Unbiased stereological estimation of the number of neurons in the human hippocampus. J Comp Neurol 296:1-22. CrossRef Medline 